\title{
Sequential Extended S-Metric Spaces and Relevant Fixed Point Results with Application to Nonlinear Integral Equations
}

\author{
Abdollah Karami, ${ }^{1}$ Shaban Sedghi $\mathbb{D}^{1},{ }^{1}$ and Vahid Parvaneh $\mathbb{D}^{2}$ \\ ${ }^{1}$ Department of Mathematics, Qaemshahr Branch, Islamic Azad University, Qaemshahr, Iran \\ ${ }^{2}$ Department of Mathematics, Gilan-E-Gharb Branch, Islamic Azad University, Gilan-E-Gharb, Iran \\ Correspondence should be addressed to Shaban Sedghi; sedghi.gh@qaemiau.ac.ir
}

Received 16 March 2021; Revised 3 May 2021; Accepted 15 May 2021; Published 21 June 2021

Academic Editor: Sergey Shmarev

Copyright (c) 2021 Abdollah Karami et al. This is an open access article distributed under the Creative Commons Attribution License, which permits unrestricted use, distribution, and reproduction in any medium, provided the original work is properly cited.

In this paper, the notion of sequential $\varsigma_{p}$-metric spaces has been introduced as a generalization of usual $S$-metric spaces, $S_{b}$-metric spaces, $S^{J S}$ metric spaces, and specially of $S_{p}$-metric spaces. In view of this notion, we prove some fixed point theorems for some classes of $\varsigma_{p}$-rational Geraghty JS-contractions over such spaces. A supporting example and an application have been given in order to examine the validity of the obtained results.

\section{Introduction and Preliminaries}

There is a huge number of extensions of Banach contraction principle. Some of them focus on using different forms of contractive conditions and some of them focus on the various generalized metric spaces. There are many interesting generalization of metric type spaces such as $b$-metric space [1], extended $b$-metric space [2], $p$-metric space [3], $J S$-metric spaces [4], $C^{*}$-algebra valued metric space [5], $S$-metric space, $S_{b}$-metric space $[6,7], S_{p}$-metric space [8], and G-metric spaces [9]. Also, for considering and analyzing more generalization of the concept of metric spaces, one can consider the following works dealing with controlled metric spaces and generalized $b$-metric spaces $[10,11]$. In the context of various metric type spaces which are the combination of the above mentioned spaces, several authors have proved different types of fixed point theorems [12]. Now, we present some definitions of some generalized metric spaces which are pertinent to our research.

Let $\Omega$ be the class of all strictly increasing continuous functions $\omega:[0, \infty) \rightarrow[0, \infty)$ with $\omega^{-1}(t) \leq t \leq \omega(t)$ and $\omega^{-1}$ $(0)=0=\omega(0)$.
Definition 1 (see [3]). Let $\mathcal{O}$ be a nonempty set. A function $p: \mathcal{O} \times \mathscr{O} \rightarrow[0, \infty)$ is a $p$-metric if for some $\omega \in \Omega$ and for all $\kappa, \kappa^{\prime}, \kappa^{\prime \prime} \in \mathcal{O}$,

(1) $p\left(\kappa, \kappa^{\prime}\right)=0$ if and only if $\kappa=\kappa^{\prime}$

(2) $p\left(\kappa, \kappa^{\prime}\right)=p\left(\kappa^{\prime}, \kappa\right)$

(3) $p\left(\kappa, \kappa^{\prime \prime}\right) \leq \omega\left(p\left(\kappa, \kappa^{\prime}\right)+p\left(\kappa^{\prime}, \kappa^{\prime \prime}\right)\right)$ for all $\kappa, \kappa^{\prime}, \kappa^{\prime \prime} \in \mathcal{O}$

In this case, the pair $(\mathcal{O}, p)$ is called a $p$-metric space, or an extended $b$-metric space.

A $b$-metric [1] is a $p$-metric with $\omega(t)=s t$, for some fixed $s \geq 1$. A $p$-metric space reduces to an ordinary metric space provided that $\omega(t)=t$.

Let $\Lambda$ be a nonempty set and $\mathscr{J} \mathcal{S}: \Lambda \times \Lambda \rightarrow[0, \infty)$ be a mapping. For any $\kappa \in \Lambda$, let us define the set

$$
C(\mathscr{J} \mathcal{S}, \Lambda, \kappa)=\left\{\left\{\kappa_{n}\right\} \subset \Lambda: \lim _{n \rightarrow \infty} \mathscr{J} \mathcal{S}\left(\kappa_{n}, \kappa\right)=0\right\}
$$

Definition 2 (JS-metric space) (see [4]). Let $\mathscr{J} \mathcal{S}: \Lambda \times \Lambda \rightarrow$ $[0, \infty]$ be a mapping so that 
(1) $\mathscr{J} \mathcal{S}\left(\kappa, \kappa^{\prime}\right)=0$ implies $\kappa=\kappa^{\prime}$

(2) for every $\kappa, \kappa^{\prime} \in \Lambda$, we have $\mathscr{J} \mathcal{S}\left(\kappa, \kappa^{\prime}\right)=\mathscr{J} \mathcal{S}\left(\kappa^{\prime}, \kappa\right)$

(3) if $\left(\kappa, \kappa^{\prime}\right) \in \Lambda \times \Lambda$ and $\left\{\kappa_{n}\right\} \in C(\mathscr{J} \mathcal{S}, \Lambda, \kappa)$ then $\mathscr{J} \mathcal{S}\left(\kappa, \kappa^{\prime}\right) \leq p \limsup \mathscr{J} \mathcal{S}\left(\kappa_{n}, \kappa^{\prime}\right)$, for some $p>0$

The pair $(\Lambda, \mathscr{J} \mathcal{S})$ is called a generalized metric space, usually known as $J S$-metric space.

In [13], Roy et al. introduced a new type of extended $b$ -metric spaces. First, let $C\left(\sigma_{p}, \Lambda, \kappa\right)$, be defined as above, where $\mathscr{J} \mathcal{S}$ is substituted by $\sigma_{p}$.

Definition 3 (see [13]). Let $\Lambda$ be a nonempty set. A mapping $\sigma_{p}: \Lambda \times \Lambda \rightarrow[0, \infty)$ is said to be a sequential $p$-metric if for all $a, b \in \Lambda$ :

(a) $\sigma_{p}(a, b)=0$ implies $a=b$

(b) $\sigma_{p}(a, b)=\sigma_{p}(b, a)$

(c) $\sigma_{p}(a, b) \leq \omega\left(\limsup \sigma_{p}\left(a_{n}, b\right)\right)$, where $\left\{a_{n}\right\} \in C\left(\sigma_{p}, \Lambda\right.$ , a) and $\omega \in \Omega$ so that $\omega^{-1}(s)=s=\omega(s)$ for $s \in\{0, \infty\}$

The triplet $\left(\Lambda, \sigma_{p}, \omega\right)$ is called a sequential $p$-metric space. We represent a sequential $p$-metric space simply as $\left(\Lambda, \sigma_{p}\right)$.

Example 4 (see [13]). Let $\Lambda=\mathbb{N}$ and the metric $\sigma_{p}: \Lambda^{2} \rightarrow[0$ $, \infty)$ be defined by

$$
\begin{cases}\sigma_{p}(1,1)=0 ; & \text { for } n \geq 2 ; \\ \sigma_{p}(n, n)=e-1, & \text { for } n \geq 2 ; \\ \sigma_{p}(1, n)=\sigma_{p}(n, 1)=e^{1 / n+1}-1, & \text { for } \text { all } n, m \geq 2 \text { with } n \neq m .\end{cases}
$$

$\sigma_{p}$ is a sequential $p$-metric on $\Lambda$ for $\omega_{1}(t)=t$ for all $t \geq 0$ and $\omega_{2}(t)=e^{t}-1$ for all $t \geq 0$.

Proposition 5 (see [13]). If $(\Lambda, \mathcal{J} \mathcal{S})$ is a JS-metric space, then $\mathcal{J} \mathcal{S}$ is also a sequential p-metric on $\Lambda$.

Proposition 6 (see [13]). Let $(\Lambda, \mathcal{J} \mathcal{S})$ be a JS-metric space with coefficient $p \geq 1$. Let $\sigma_{p}(a, b):=\Gamma(\mathscr{J} \mathcal{S}(a, b))$, where $\Gamma \in \Omega$. Then, $\sigma_{p}$ is a sequential p-metric with $\omega(t)=\Gamma(p t)$ for all $t \geq 0$.

Sedghi et al. [14] have recommended the notion of a $S$ -metric space as follows.
Definition 7 (see [14]). Let $\mathcal{O}$ be a nonempty set. An $S$-metric on $\mathcal{O}$ is a function $S: \mathcal{O} \times \mathcal{O} \times \mathcal{O} \rightarrow[0, \infty)$ such that,

$$
S\left(\kappa, \kappa^{\prime}, \kappa^{\prime \prime}\right) \geq 0, \text { and }
$$

(1) $S\left(\kappa, \kappa^{\prime}, \kappa^{\prime \prime}\right)=0$ if and only if $\kappa=\kappa^{\prime}=\kappa^{\prime \prime}$

(2) $S\left(\kappa, \kappa^{\prime}, \kappa^{\prime \prime}\right) \leq S(\kappa, \kappa, a)+S\left(\kappa^{\prime}, \kappa^{\prime}, a\right)+S\left(\kappa^{\prime \prime}, \kappa^{\prime \prime}, a\right)$ for all $\kappa, \kappa^{\prime}, \kappa^{\prime \prime}, a \in \mathcal{O}$

for each $\kappa, \kappa^{\prime}, \kappa^{\prime \prime}, a \in \mathcal{O}$.

The pair $(\mathcal{O}, S)$ is nominated an $S$-metric space.

Some examples of such $S$-metric spaces are

(1) Let $\mathcal{O}$ be a normed space. Then, $S\left(\kappa, \kappa^{\prime}, \kappa^{\prime \prime}\right)=\| \kappa^{\prime}+$ $\kappa^{\prime \prime}-2 \kappa\|+\| \kappa^{\prime}-\kappa^{\prime \prime} \|$ is an $S$-metric on $\mathcal{O}$

(2) Let $\mathcal{O}$ be a normed space. Then, $S\left(\kappa, \kappa^{\prime}, \kappa^{\prime \prime}\right)=\| \kappa-\kappa$ $"\|+\| \kappa^{\prime}-\kappa^{\prime \prime} \|$ is an $S$-metric on $\mathcal{O}$

(3) Let $\mathcal{O}$ be a nonempty set and $d$ be an ordinary metric on 0 . Then, $S\left(\kappa, \kappa^{\prime}, \kappa^{\prime \prime}\right)=d\left(\kappa, \kappa^{\prime}\right)+d\left(\kappa^{\prime}, \kappa^{\prime \prime}\right)$ is an $S$ -metric on $\mathcal{O}$

Lemma 8 (see [14]). In an $S$-metric space, we have $S(\kappa$ $\left., \kappa, \kappa^{\prime}\right)=S\left(\kappa^{\prime}, \kappa^{\prime}, \kappa\right)$.

Definition 9 (see [8]). Let $\mathcal{O}$ be a nonempty set and $\omega \in \Omega$. Suppose that a mapping $\varsigma_{p}: \mathcal{O} \times \mathscr{O} \times \mathscr{O} \rightarrow[0, \infty)$ satisfies:

(1) $\varsigma_{p}\left(\kappa, \kappa^{\prime}, \kappa^{\prime \prime}\right)=0$ iff $\kappa=\kappa^{\prime}=\kappa^{\prime \prime}$

(2) $\varsigma_{p}\left(\kappa, \kappa^{\prime}, \kappa^{\prime \prime}\right) \leq \omega\left(\varsigma_{p}(\kappa, \kappa, a)+\varsigma_{p}\left(\kappa^{\prime}, \kappa^{\prime}, a\right)+\varsigma_{p}\left(\kappa^{\prime \prime}, \kappa^{\prime \prime}\right.\right.$ ,a)) for all $\kappa, \kappa^{\prime}, \kappa^{\prime \prime}, a \in \mathcal{O}$

Then $\varsigma_{p}$ is called a modified $S$-metric, and the pair $\left(\mathcal{O}, \varsigma_{p}\right)$ is called a modified $S$-metric space.

An $S$-metric space is an $\varsigma_{p}$-metric space where $\omega(t)=t$ and every $S_{b}$-metric space with parameter $s \geq 1$ is an $\varsigma_{p}$ -metric space where $\omega(t)=s t$.

Definition 10. A $\varsigma_{p}$-metric is called symmetric if $\varsigma_{p}\left(\kappa, \kappa, \kappa^{\prime}\right)$ $=\varsigma_{p}\left(\kappa^{\prime}, \kappa^{\prime}, \kappa\right)$, for all $\kappa, \kappa^{\prime} \in \mathcal{O}$.

Example 11 (see [8]). Let $(\mathcal{O}, S)$ be an $S$-metric space. Then

(1) $\varsigma_{p}\left(\kappa, \kappa^{\prime}, \kappa^{\prime \prime}\right)=e^{S\left(\kappa, \kappa^{\prime}, \kappa^{\prime \prime}\right)}-1$ is an $\varsigma_{p}$-metric with $\omega(t)$ $=e^{t}-1$

(2) $\varsigma_{p}\left(\kappa, \kappa^{\prime}, \kappa^{\prime \prime}\right)=S\left(\kappa, \kappa^{\prime}, \kappa^{\prime \prime}\right) e^{S\left(\kappa, \kappa^{\prime}, \kappa^{\prime \prime}\right)}$ is an $\varsigma_{p}$-metric with $\omega(t)=t e^{t}$ 
In general, an $\varsigma_{p}$-metric with nontrivial function $\omega$ need not to be jointly continuous in all its variables (see [15]). The following simple lemma is a modification of Lemma 1.11 of [8].

Lemma 12. Let $\left(\mathcal{O}, \varsigma_{p}\right)$ be an $\varsigma_{p}$-metric space.

(1) Suppose that $\left\{\kappa_{n}\right\}$ and $\left\{\kappa_{n}^{\prime}\right\}$ are $\varsigma_{p}$-convergent to $\kappa$ and $\kappa^{\prime}$, respectively. Then, we have

$$
\begin{aligned}
& \omega^{-2}\left(\varsigma_{p}\left(\kappa, \kappa, \kappa^{\prime}\right)\right) \leq \liminf _{n \rightarrow \infty} \varsigma_{p}\left(\kappa_{n}, \kappa_{n}, \kappa_{n}^{\prime}\right) \\
& \quad \leq \limsup _{n \rightarrow \infty} \varsigma_{p}\left(\kappa_{n}, \kappa_{n}, \kappa_{n}^{\prime}\right) \leq \omega^{2}\left(\varsigma_{p}\left(\kappa, \kappa, \kappa^{\prime}\right)\right) .
\end{aligned}
$$

In particular, if $\kappa=\kappa^{\prime}$, then, we have $\lim _{n \rightarrow \infty} \varsigma_{p}\left(\kappa_{n}, \kappa_{n}, \kappa_{n}^{\prime}\right)=0$

(2) Suppose that $\varsigma_{p}$-metric $\left(\mathcal{O}, \varsigma_{p}\right)$ is symmetric and $\left\{\kappa_{n}\right\}$ is $\varsigma_{p}$-convergent to $\kappa$ and $\kappa^{\prime \prime} \in \mathcal{O}$ is arbitrary. Then, we have

$$
\begin{aligned}
& \omega^{-1}\left(\varsigma_{p}\left(\kappa, \kappa, \kappa^{\prime \prime}\right)\right) \leq \liminf _{n \rightarrow \infty} \varsigma_{p}\left(\kappa_{n}, \kappa_{n}, \kappa^{\prime \prime}\right) \\
& \quad \leq \limsup _{n \rightarrow \infty} \varsigma_{p}\left(\kappa_{n}, \kappa_{n}, \kappa^{\prime \prime}\right) \leq \omega\left(\varsigma_{p}\left(\kappa, \kappa, \kappa^{\prime \prime}\right)\right) .
\end{aligned}
$$

Proof. (1) Using the rectangle inequality in the $\varsigma_{p}$-metric space, it is easy to see that

$$
\begin{aligned}
& \varsigma_{p}\left(\kappa, \kappa, \kappa^{\prime}\right) \leq \omega\left[2 \varsigma_{p}\left(\kappa, \kappa, \kappa_{n}\right)+\varsigma_{p}\left(\kappa^{\prime}, \kappa^{\prime}, \kappa_{n}\right)\right] \\
& \quad \leq \omega\left[2 \varsigma_{p}\left(\kappa, \kappa, \kappa_{n}\right)+\omega\left[2 \varsigma_{p}\left(\kappa^{\prime}, \kappa^{\prime}, \kappa_{n}^{\prime}\right)+\varsigma_{p}\left(\kappa_{n}, \kappa_{n}, \kappa_{n}^{\prime}\right)\right]\right], \\
& \varsigma_{p}\left(\kappa_{n}, \kappa_{n}, \kappa_{n}^{\prime}\right) \leq \omega\left[2 \varsigma_{p}\left(\kappa_{n}, \kappa_{n}, \kappa\right)+\varsigma_{p}\left(\kappa_{n}^{\prime}, \kappa_{n}^{\prime}, \kappa\right)\right] \\
& \quad \leq \omega\left[2 \varsigma_{p}\left(\kappa_{n}, \kappa_{n}, \kappa\right)+\omega\left[2 \varsigma_{p}\left(\kappa_{n}^{\prime}, \kappa_{n}^{\prime}, \kappa^{\prime}\right)+\varsigma_{p}\left(\kappa, \kappa, \kappa^{\prime}\right)\right]\right] .
\end{aligned}
$$

The desired result is obtained via letting $n \rightarrow \infty$ in the above inequalities and taking lower limit and the upper limit in the first and second inequality, respectively.

(2) Using the rectangle inequality, we see that

$$
\begin{aligned}
& \omega^{-1}\left(\varsigma_{p}\left(\kappa, \kappa, \kappa^{\prime \prime}\right)\right) \leq 2 \varsigma_{p}\left(\kappa, \kappa, \kappa_{n}\right)+\varsigma_{p}\left(\kappa_{n}, \kappa_{n}, \kappa^{\prime \prime}\right), \\
& \varsigma_{p}\left(\kappa_{n}, \kappa_{n}, \kappa^{\prime \prime}\right) \leq \omega\left(2 \varsigma_{p}\left(\kappa_{n}, \kappa_{n}, \kappa\right)+\varsigma_{p}\left(\kappa^{\prime \prime}, \kappa^{\prime \prime}, \kappa\right)\right) .
\end{aligned}
$$

The desired result is obtained via taking liminf as $n \rightarrow \infty$ in the first inequality and the limsup as $n \rightarrow \infty$ in the second inequality, respectively.

\section{Introduction to Sequential $\varsigma_{p}$-Metric Spaces}

In this section, we introduce a new type of extended $\varsigma_{p}$-metric spaces. First, let us to define

$$
C\left(\varsigma_{p}, \mathcal{O}, a\right)=\left\{\left\{a_{n}\right\} \subset \mathcal{O}: \lim _{n \rightarrow \infty} \varsigma_{p}\left(a, a, a_{n}\right)=0\right\},
$$

where $\varsigma_{p}: \mathcal{O} \times \mathcal{O} \times \mathcal{O} \rightarrow[0, \infty]$ is a given mapping and $\mathscr{O}$ is a nonempty set.

Definition 13. Let $\mathcal{O}$ be a nonempty set. A mapping $\varsigma_{p}: \mathcal{O} \times$ $\mathscr{O} \times \mathcal{O} \rightarrow[0, \infty]$ is said to be a sequential $\varsigma_{p}$-metric if for all $a, b, c \in \mathcal{O}:$

(1) $\varsigma_{p}(a, b, c)=0$ implies $a=b=c$

(2) $\varsigma_{p}(a, b, c) \leq \omega\left(\limsup _{n \rightarrow \infty} \varsigma_{p}\left(a, a, c_{n}\right)+\limsup _{n \rightarrow \infty} \varsigma_{p}\right.$ $\left.\left(b, b, c_{n}\right)\right)$, where $\left\{c_{n}\right\} \in C\left(\varsigma_{p}, \mathcal{O}, c\right)$ and $\omega \in \Omega$ so that $\omega^{-1}(s)=s=\omega(s)$ for $s \in\{0, \infty\}$

The triplet $\left(\mathcal{O}, \varsigma_{p}, \omega\right)$ is called a sequential $\varsigma_{p}$-metric space (SSPMS). It will be shown simply as $\left(\mathcal{O}, \varsigma_{p}\right)$.

The above definition is an extension of Definition 13 of [13], that is, the definition of $S^{J S}$-metric spaces.

Example 14. Let the triplet $\left(\Lambda, \sigma_{p}, \omega\right)$ be a sequential $p$-metric space. If we define $\varsigma_{p}\left(\kappa, \kappa^{\prime}, \kappa^{\prime \prime}\right)=\sigma_{p}\left(\kappa, \kappa^{\prime}\right)+\sigma_{p}\left(\kappa^{\prime}, \kappa^{\prime \prime}\right)$, then, $\varsigma_{p}$ will be a sequential $\varsigma_{p}$-metric with the same control function $\omega$ provided the $\omega$ is subadditive.

According to the above example and Example 15, we construct the following example of $\varsigma_{p}$-metrics.

Example 15. Let $\Lambda=\mathbb{N}$ and the metric $\varsigma_{p}: \Lambda^{3} \rightarrow[0, \infty)$ be defined by

$$
\begin{cases}\varsigma_{p}(1,1,1)=0 ; & \text { for } n \geq 2 ; \\ \varsigma_{p}(n, n, n)=2 e-2, & n \geq 2 ; \\ \varsigma_{p}(1, n, n)=\varsigma_{p}(1,1, n)=\varsigma_{p}(n, n, 1)=\varsigma_{p}(n, 1,1)=e^{1 / n+1}+e-2, & n \geq 2 ; \\ \varsigma_{p}(n, 1, n)=\varsigma_{p}(1, n, 1)=2 e^{1 / n+1}-2, & m, n \geq 2, m \neq n, \\ \varsigma_{p}(n, n, m)=\varsigma_{p}(m, n, n)=\varsigma_{p}(n, m, m)=\varsigma_{p}(m, m, n)=e^{m n}+e-2, & m, n \geq 2, m \neq n, \\ \varsigma_{p}(m, n, m)=\varsigma_{p}(n, m, n)=2 e^{m n}-2, & m, n, o \geq 2, n \neq m . \\ \varsigma_{p}(m, n, o)=e^{m n}-2+e^{n o}-2, & \end{cases}
$$

$\varsigma_{p}$ is a sequential $p$-metric on $\Lambda$ for $\omega_{1}(t)=t$.

Proposition 16. Let $\left(\mathcal{O}, \varsigma_{p}\right)$ be a $S^{J S}$-metric space with coefficient $k \geq 1$. Let $\varsigma_{p}^{\prime}(a, b, c)=\Gamma\left(\varsigma_{p}(a, b, c)\right)$, where $\Gamma \in \Omega$. Then, $\varsigma_{p}^{\prime}$ is a sequential $\varsigma_{p}$-metric for $\omega(t)=\Gamma(k t)$ for all $a, b, c \in \mathcal{O}$ and $t \geq 0$. 
Proof. Here, we show that $\left(\mathcal{O}, \varsigma_{p}^{\prime}\right)$ satisfies all the conditions of Definition 25.

(a) $\varsigma_{p}^{\prime}(a, b, c)=0$ gives $\Gamma\left(\varsigma_{p}(a, b, c)\right)=0$. Then $\varsigma_{p}(a, b, c)$ $=\Gamma^{-1}(0)=0$ which implies $a=b=c$

(b) For all $a, b, c \in \mathcal{O}$, we have $\varsigma_{p}^{\prime}(a, b, c)=\Gamma\left(\varsigma_{p}(a, b, c)\right) \leq$ $\Gamma\left(k \limsup _{n \rightarrow \infty} \varsigma_{p}\left(a, a, c_{n}\right)+k \limsup _{n \rightarrow \infty} \varsigma_{p}\left(b, b, c_{n}\right)\right)$ $=\omega\left(\limsup _{n \rightarrow \infty} \varsigma_{p}\left(a, a, c_{n}\right)+\limsup _{n \rightarrow \infty} \varsigma_{p}\left(b, b, c_{n}\right)\right)$ where $\left\{c_{n}\right\} \in C\left(\varsigma_{p}, \mathcal{O}, c\right)=C\left(\varsigma_{p}^{\prime}, \mathcal{O}, c\right)$.

Proposition 17. Let $\left(\mathcal{O}, \varsigma_{p}\right)$ be a SSPMS with control function $\omega$. Let $\varsigma_{p}^{\prime}(a, b, c)=\Gamma\left(\varsigma_{p}(a, b, c)\right)$, where $\Gamma \in \Omega$. Then, $\varsigma_{p}^{\prime}$ is a sequential $\varsigma_{p}$-metric with $\omega^{\prime}(t)=\Gamma(\omega(t))$ for all $t \geq 0$.

Proof. Here, we show that $\left(\mathcal{O}, \varsigma_{p}^{\prime}\right)$ satisfies all the conditions of Definition 25.

(a) $\varsigma_{p}^{\prime}(a, b, c)=0$ gives $\Gamma\left(\varsigma_{p}(a, b, c)\right)=0$. Then $\varsigma_{p}(a, b, c)$ $=\Gamma^{-1}(0)=0$ which implies $a=b=c$

(b) For all $a, b, c \in \mathcal{O}$, we have $\varsigma_{p}^{\prime}(a, b, c)=\Gamma\left(\varsigma_{p}(a, b, c)\right)$ $\leq \Gamma\left(\omega\left(\limsup _{n \rightarrow \infty} \varsigma_{p}\left(a, a, c_{n}\right)\right)+\omega\left(\limsup _{n \rightarrow \infty} \varsigma_{p}(b\right.\right.$, $\left.\left.\left.b, c_{n}\right)\right)\right)$ where $\left\{c_{n}\right\} \in C\left(\varsigma_{p}, \mathcal{O}, c\right)=C\left(\varsigma_{p}^{\prime}, \mathcal{O}, c\right)$.

Definition 18. Let $\left(\mathcal{O}, \varsigma_{p}\right)$ be a SSPMS. Also, let $\left\{a_{n}\right\}$ be a sequence in $\mathcal{O}$ and $a \in \mathcal{O}$.

(i) $\left\{a_{n}\right\}$ is said to be convergent and converges to $a$ if $\left\{a_{n}\right\} \in C\left(s_{p}, \mathcal{O}, a\right)$,

(ii) $\left\{a_{n}\right\}$ is said to be Cauchy if $\lim _{n, m \rightarrow \infty} \varsigma_{p}\left(a_{n}, a_{n}, a_{m}\right)$ $=0$

(iii) $\mathcal{O}$ is said to be complete if any Cauchy sequence in $\mathcal{O}$ is convergent

Definition 19 (see [15]). Let $\left(\mathcal{O}, \varsigma_{p}\right)$ and $\left(\mathfrak{D}^{\prime}, \varsigma_{p}^{*}\right)$ be two sequential $\varsigma_{p}$-metric spaces. A mapping $\mathscr{H}: \mathcal{O} \rightarrow \mathfrak{D}^{\prime}$ is called continuous at a point $a \in \mathcal{O}$ if for any $\varepsilon>0$ there exists $\delta_{\varepsilon}>0$ such that for any $l \in \mathcal{O}, \varsigma_{p}^{*}(\mathscr{H} l, \mathscr{H} l, \mathscr{H} a)<\varepsilon$ whenever $\varsigma_{p}(l, l$ , a) $<\delta_{\varepsilon} . \mathscr{H}$ is said to be continuous on $\mathcal{O}$ if $\mathscr{H}$ is continuous at each Point of $\mathcal{O}$.

Proposition 20. In a SSPMS $\left(\mathcal{O}, \varsigma_{p}\right)$ if a sequence $\left\{a_{n}\right\}$ is convergent, then, it converges to unique element in $\mathcal{O}$.

Proposition 21. Let $\left(\mathcal{O}, \varsigma_{p}\right)$ be a SSPMS and $\left\{a_{n}\right\} \subset \mathcal{O}$ converges to some $a \in \mathcal{O}$. Then, $\varsigma_{p}(a, a, a)=0$.

Proof. Since $\left\{a_{n}\right\}$ converges to $a \in \mathcal{O}$, so $\lim _{n \rightarrow \infty} \varsigma_{p}\left(a_{n}, a_{n}, a\right)$ $=0$. Therefore, we have $\varsigma_{p}(a, a, a) \leq \omega\left(2 \limsup _{n \rightarrow \infty} \varsigma_{p}(a, a\right.$, $\left.\left.a_{n}\right)\right)=\omega(0)=0$ which implies $\varsigma_{p}(a, a, a)=0$.
Proposition 21. Let $\left\{a_{n}\right\}$ be a Cauchy sequence in a SSPMS $\left(\mathcal{O}, \varsigma_{p}, \omega\right)$ such that $\omega^{-1}$ is continuous. If $\left\{a_{n}\right\}$ has a convergent subsequence $\left\{a_{n_{k}}\right\}$ which converges to an $a \in \mathcal{O}$, then, $\left\{a_{n}\right\}$ also converges to $a \in \mathcal{O}$.

Proof. From condition (3) of Definition 25, we have

$$
\varsigma_{p}\left(a, a, a_{n}\right) \leq \omega\left(\limsup _{k \rightarrow \infty} \varsigma_{p}\left(a_{n_{k}}, a_{n}, a_{n}\right)\right)
$$

which implies that

$$
\omega^{-1}\left(\varsigma_{p}\left(a, a, a_{n}\right)\right) \leq \limsup _{k \rightarrow \infty} \varsigma_{p}\left(a_{n_{k}}, a_{n}, a_{n}\right)
$$

for all $n \in \mathbb{N}$. Due to the Cauchyness of $\left\{a_{n}\right\}$, it follows that $\lim _{n, k \rightarrow \infty} \varsigma_{p}\left(a_{n_{k}}, a_{n}, a_{n}\right)=0$ and thus $\omega^{-1}\left(\varsigma_{p}\left(a, a, a_{n}\right)\right) \rightarrow 0$ as $n \rightarrow \infty$ which implies that $\varsigma_{p}\left(a, a, a_{n}\right) \rightarrow 0$ as $n \rightarrow \infty$, since $\omega^{-1}$ is continuous. Hence, $\left\{a_{n}\right\}$ converges to $a \in \mathcal{O}$.

Proposition 22. In a SSPMS $\left(\mathcal{O}, \varsigma_{p}\right)$ if a self mapping $\mathscr{H}$ is continuous at $a \in \mathcal{O}$, then $\left\{\mathscr{H} a_{n}\right\} \in C\left(\varsigma_{p}, \mathcal{O}, \mathscr{H} a\right)$ for any sequence $\left\{a_{n}\right\} \in C\left(\varsigma_{p}, \mathcal{O}, a\right)$.

Proof. Let $\varepsilon>0$ be given. Since $\mathscr{H}$ is continuous at $a$, then for any $\varepsilon>0$ there exists $\delta_{\varepsilon}>0$ such that $\varsigma(c, c, a)<\delta_{\varepsilon}$ implies $\varsigma$ $\left(\mathscr{H}_{c}, \mathscr{H}_{c}, \mathscr{H}_{a}\right)<\varepsilon$. As $\left\{a_{n}\right\}$ converges to $a$, so for $\delta_{\varepsilon}>0$, there exists $N \in \mathbb{N}$ such that $\varsigma\left(a, a, a_{n}\right)<\delta_{\varepsilon}$ for all $n \geq N$. Therefore, for any $n \geq N, \varsigma\left(\mathscr{H} a, \mathscr{H} a, \mathscr{H} a_{n}\right)<\varepsilon$ and thus $\mathscr{H}$ $a_{n} \rightarrow \mathscr{H}$ a as $n \rightarrow \infty$.

Definition 23. Let $\left(\mathcal{O}, \varsigma_{p}\right)$ be a SSPMS with supporting function $\omega$. Define

$$
\begin{aligned}
B_{p}(a, \varepsilon) & :=\left\{b \in \mathcal{O}: \varsigma_{p}(a, a, b)<\varsigma_{p}(a, a, a)+\varepsilon\right\}, \\
B_{p}[a, \varepsilon]: & =\left\{b \in \mathcal{O}: \varsigma_{p}(a, a, b) \leqslant \varsigma_{p}(a, a, a)+\varepsilon\right\},
\end{aligned}
$$

for all $a \in \mathcal{O}$ and $\varepsilon>0$.

Remark 24. One can easily check that the collection

$\left.\tau_{c_{p}}:=\{\varnothing\} \cup U(\neq \varnothing)\right) \subset \mathcal{O}:$ for any $a \in U$ there exists $\varepsilon>0: B_{p}(a, \varepsilon) \subset U$,

forms a topology on $\mathcal{O}$.

Another approach to define a sequential $\varsigma_{p}$-metric is as follows:

Define

$$
C\left(\varsigma_{p}, \mathcal{O}, a\right)=\left\{\left\{a_{n}\right\} \subset \mathcal{O}: \lim _{n \rightarrow \infty} \varsigma_{p}\left(a_{n}, a_{n}, a\right)=0\right\}
$$

where $\varsigma_{p}: \mathcal{O} \times \mathcal{O} \times \mathcal{O} \rightarrow[0, \infty]$ is a given mapping, and $\mathcal{O}$ is a nonempty set. 
Definition 25. Let $\mathcal{O}$ be a nonempty set. A mapping $\varsigma_{p}: \mathcal{O} \times$ $\mathcal{O} \times \mathcal{O} \rightarrow[0, \infty]$ is said to be a sequential $\varsigma_{p}$-metric if for all $a, b, c \in \mathcal{O}$ :

(1) $\varsigma_{p}(a, b, c)=0$ implies $a=b=c$

(2) $\varsigma_{p}(a, b, c) \leq \omega\left(\limsup _{n \rightarrow \infty} \varsigma_{p}\left(c_{n}, c_{n}, a\right)+\limsup _{n \rightarrow \infty}\right.$ $\left.\varsigma_{p}\left(c_{n}, c_{n}, b\right)\right)$, where $\left\{c_{n}\right\} \in C\left(\varsigma_{p}, \mathcal{O}, c\right)$ and $\omega \in \Omega$ and $\omega^{-1}(s)=s=\omega(s)$ for $s \in\{0, \infty\}$

The triplet $\left(\mathcal{O}, \varsigma_{p}, \omega\right)$ is called a sequential $\varsigma_{p}$-metric space. It will be shown simply as $\left(\mathcal{O}, \varsigma_{p}\right)$.

In this paper, we investigate the existence of unique fixed point for some rational contractions of Jleli-Samet and Geraghty type in ordered sequential $\varsigma_{p}$-spaces. Our motivation is an interesting generalization of Banach contraction principle which is presented by Jleli et al. in [16].

\section{Main Results}

Note that $(\mathcal{O}, \preceq)$ possesses the s.l.c.p. whenever $\left\{\kappa_{n}\right\}$ is a nondecreasing sequence in $\mathcal{O}$ such that $\kappa_{n} \rightarrow \mathfrak{U} \in \mathcal{O}$, one has $\kappa_{n}$ $\preceq \mathfrak{U}$ for all $n \in \mathbb{N}$. From now on, by SSPMS, we mean a sequential $\varsigma_{p}$-metric space and by SPRGJSC a $\varsigma_{p}$-rational Geraghty JS-contraction.
Let $\mathscr{G}$ denotes the class of all functions $(\mathfrak{S}:[0, \infty) \rightarrow[0$, $\left.\omega^{-1}(1)\right)$ satisfying the following condition:

$$
\underset{n \rightarrow \infty}{\limsup } \mathfrak{G}\left(t_{n}\right)=\omega^{-1}(1) \text { implies that } t_{n} \rightarrow 0 \text {, asn } \rightarrow \infty \text {. }
$$

The following are two examples of Geraghty functions $\mathfrak{G}$.

(1) $\mathfrak{G}(t)=e^{-t^{p} t^{t^{q}}}$ for all $t>0$ and $\mathfrak{G}(t)=0$ for $t=0$, where $p, q>0$

(2) $\mathfrak{G}(t)=1 /\left(1+t^{p}\right)$ for all $t>0$ and $\mathfrak{G}(t)=0$ for $t=0$, where $p>0$

In the above examples, we assumed that $\omega(t)=t$. In the case that, for instance, $\omega(t)=\sinh t$, then $\omega^{-1}(1) \simeq 0.88$, and one can multiple this amount to the above functions $\omega$.

Definition 26. Let $\left(\mathcal{O}, \varsigma_{p}\right)$ be an ordered SSPMS. A mapping Q : $\mathcal{O} \rightarrow \mathcal{O}$ is called a SPRGJSC of type $I$ if

$$
\theta\left(\varsigma_{p}\left(\widehat{Q} \kappa, Q \mathcal{Q} \kappa^{\prime}, \mathcal{Q} \kappa^{\prime \prime}\right)\right) \leq \theta\left(M\left(\kappa, \kappa^{\prime}, \kappa^{\prime \prime}\right)\right)^{\mathfrak{G}\left(M\left(\kappa, \kappa^{\prime}, \kappa^{\prime \prime}\right)\right)},
$$

for some $\mathscr{G} \in \mathscr{G}$ and for all comparable elements $\kappa$, $\kappa^{\prime}, \kappa^{\prime \prime} \in \mathcal{O}$, where

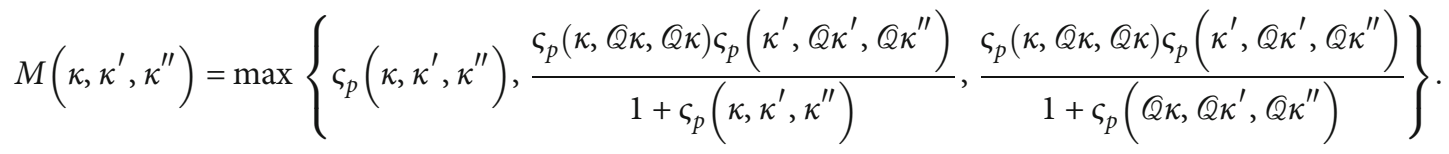

Theorem 27. Let $\left(\mathcal{O}, \preceq, \varsigma_{p}, \omega\right)$ be an ordered $\varsigma_{p}$-complete SSPMS. Let $\mathbb{Q}: \mathcal{O} \rightarrow \mathcal{O}$ be $a \preceq$-increasing mapping such that $\kappa_{0} \preceq Q\left(\kappa_{0}\right)$ for some element $\kappa_{0} \in \mathcal{O}$. Suppose that $\mathbb{Q}$ be a SPRGJSC of type I. If

(I) Q is continuous, or

(II) $(\mathcal{O}, \preceq)$ possesses the s.l.c.p

then Q has a fixed point. Moreover, the set of fixed points of $Q$ is well ordered if and only if $F(Q)$ is a singleton.

Proof. Put $\kappa_{n}=\mathbb{Q}^{n}\left(\kappa_{0}\right)$. Since $\kappa_{0} \preceq Q\left(\kappa_{0}\right)$ and $\mathbb{Q}$ is $\preceq$-increasing, we obtain by induction that

$$
\kappa_{0} \preceq Q\left(\kappa_{0}\right) \preceq Q^{2}\left(\kappa_{0}\right) \preceq \cdots \preceq Q^{n}\left(\kappa_{0}\right) \preceq Q^{n+1}\left(\kappa_{0}\right) \preceq .
$$

We will do the proof in the following steps.

Step I. We will show that $\lim _{n \rightarrow \infty} \varsigma_{p}\left(\kappa_{n}, \kappa_{n+1}, \kappa_{n+1}\right)=0$. Without any loss of generality, we may assume that $\kappa_{n} \neq \kappa_{n+1}$, for all $n \in \mathbb{N}$. Since $\kappa_{\mathrm{n}} \leq \kappa_{n+1}$ for each $n \in \mathbb{N}$, then by (16), we have

$$
\begin{aligned}
& \theta\left(\varsigma_{p}\left(\kappa_{n}, \kappa_{n+1}, \kappa_{n+1}\right)\right)=\theta\left(\varsigma_{p}\left(\mathbb{Q} \kappa_{n-1}, \mathbb{Q} \kappa_{n}, \mathbb{Q} \kappa_{n}\right)\right) \\
& \quad \leq \theta\left(M\left(\kappa_{n-1}, \kappa_{n}, \kappa_{n}\right)\right)^{\mathfrak{G}\left(M\left(\kappa_{n-1}, \kappa_{n}, \kappa_{n}\right)\right)}
\end{aligned}
$$

because

$$
\begin{aligned}
& M\left(\kappa_{n-1}, \kappa_{n}, \kappa_{n}\right)
\end{aligned}
$$

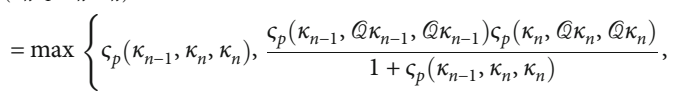

$$
\begin{aligned}
& \left.\cdot \frac{\varsigma_{p}\left(\kappa_{n-1}, \mathbb{Q} \kappa_{n-1}, \mathbb{Q} \kappa_{n-1}\right) \varsigma_{p}\left(\kappa_{n}, \mathbb{Q} \kappa_{n}, \mathbb{Q} \kappa_{n}\right)}{1+\varsigma_{p}\left(\mathbb{Q} \kappa_{n-1}, \mathbb{Q} \kappa_{n}, \mathbb{Q} \kappa_{n}\right)}\right\} \\
& =\max \left\{\varsigma_{p}\left(\kappa_{n-1}, \kappa_{n}, \kappa_{n}\right), \frac{\varsigma_{p}\left(\kappa_{n-1}, \kappa_{n}, \kappa_{n}\right) \varsigma_{p}\left(\kappa_{n}, \kappa_{n+1}, \kappa_{n+1}\right)}{1+\varsigma_{p}\left(\kappa_{n-1}, \kappa_{n}, \kappa_{n}\right)},\right. \\
& \left.\cdot \frac{\varsigma_{p}\left(\kappa_{n-1}, \kappa_{n}, \kappa_{n}\right) \varsigma_{p}\left(\kappa_{n}, \kappa_{n+1}, \kappa_{n+1}\right)}{1+\varsigma_{p}\left(\kappa_{n}, \kappa_{n+1}, \kappa_{n+1}\right)}\right\} \leq \max \left\{\varsigma_{p}\left(\kappa_{n-1}, \kappa_{n}, \kappa_{n}\right), \varsigma_{p}\left(\kappa_{n}, \kappa_{n+1}, \kappa_{n+1}\right)\right\} \text {, }
\end{aligned}
$$

and if $\max \left\{\varsigma_{p}\left(\kappa_{n-1}, \kappa_{n}, \kappa_{n}\right), \varsigma_{p}\left(\kappa_{n}, \kappa_{n+1}, \kappa_{n+1}\right)\right\}=\varsigma_{p}\left(\kappa_{n}, \kappa_{n+1}\right.$, 
$\left.\kappa_{n+1}\right)$, then from (19), we have

$$
\begin{gathered}
\theta\left(\varsigma_{p}\left(\kappa_{n}, \kappa_{n+1}, \kappa_{n+1}\right)\right) \leq \theta\left(\varsigma_{p}\left(\kappa_{n}, \kappa_{n+1}, \kappa_{n+1}\right)\right)^{\mathfrak{G}\left(M\left(\kappa_{n-1}, \kappa_{n}, \kappa_{n}\right)\right)} \\
<\theta\left(\varsigma_{p}\left(\kappa_{n}, \kappa_{n+1}, \kappa_{n+1}\right)\right)^{\omega^{-1}(1)}<\theta\left(\varsigma_{p}\left(\kappa_{n}, \kappa_{n+1}, \kappa_{n+1}\right)\right)
\end{gathered}
$$

which is a contradiction.

Hence, $\max \left\{\varsigma_{p}\left(\kappa_{n-1}, \kappa_{n}, \kappa_{n}\right), \varsigma_{p}\left(\kappa_{n}, \kappa_{n+1}, \kappa_{n+1}\right)\right\}=\varsigma_{p}($ $\left.\kappa_{n-1}, \kappa_{n}, \kappa_{n}\right)$. So, from (19),

$$
\begin{aligned}
& \theta\left(\varsigma_{p}\left(\kappa_{n}, \kappa_{n+1}, \kappa_{n+1}\right)\right) \leq \theta\left(\varsigma_{p}\left(\kappa_{n-1}, \kappa_{n}, \kappa_{n}\right)\right)^{\mathfrak{G}\left(M\left(\kappa_{n-1}, \kappa_{n}, \kappa_{n}\right)\right)} \\
& \quad<\theta\left(\varsigma_{p}\left(\kappa_{n-1}, \kappa_{n}, \kappa_{n}\right)\right) .
\end{aligned}
$$

That is, $\left\{\varsigma_{p}\left(\kappa_{n}, \kappa_{n+1}, \kappa_{n+1}\right)\right\}$ is a decreasing sequence, so, there exists $r \geq 0$ such that $\lim _{n \rightarrow \infty} \varsigma_{p}\left(\kappa_{n}, \kappa_{n+1}, \kappa_{n+1}\right)=r$. We will prove that $r=0$. Suppose on contrary that $r>0$. Then, letting $n \rightarrow \infty$, from (22), we have

$$
\theta(r) \leq \theta(r)^{\lim _{n \rightarrow \infty}\left(\mathfrak{s}\left(M\left(\kappa_{n-1}, \kappa_{n}, \kappa_{n}\right)\right)\right.}
$$

which implies that $\omega^{-1}(1) \leq 1 \leq \lim _{n \rightarrow \infty} \mathfrak{G}\left(M\left(\kappa_{n-1}, \kappa_{n}, \kappa_{n}\right)\right)$. Now, as $\mathscr{G} \in \mathscr{G}$ we conclude that $M\left(\kappa_{n-1}, \kappa_{n}, \kappa_{n}\right) \rightarrow 0$ which yields that $r=0$, a contradiction. Hence,

$$
\lim _{n \rightarrow \infty} \varsigma_{p}\left(\kappa_{n}, \kappa_{n+1}, \kappa_{n+1}\right)=0
$$

Step II. Now, we show that the sequence $\left\{\kappa_{n}\right\}$ is a $\varsigma_{p}$-Cauchy sequence. Suppose that $\left\{\kappa_{n}\right\}$ is not $\varsigma_{p}$-Cauchy. Then for some $\varepsilon>0$ we can find two subsequences $\left\{\kappa_{m_{i}}\right\}$ and $\left\{\kappa_{n_{i}}\right\}$ of $\left\{\kappa_{n}\right\}$ such that $m_{i}$ and $n_{i}$ are the smallest index such that

$$
n_{i}>m_{i}>i \text { and } \varsigma_{p}\left(\kappa_{m_{i}}, \kappa_{n_{i}}, \kappa_{n_{i}}\right) \geq \varepsilon
$$

This means that

$$
\varsigma_{p}\left(\kappa_{m_{i}-1}, \kappa_{n_{i}-1}, \kappa_{n_{i}-1}\right)<\varepsilon
$$

From the definition of $M\left(\kappa, \kappa^{\prime}, \kappa^{\prime \prime}\right)$ and the above limits,

$$
\begin{aligned}
& \limsup _{i \rightarrow \infty} M\left(\kappa_{m_{i}-1}, \kappa_{n_{i}-1}, \kappa_{n_{i}-1}\right) \\
& =\limsup _{i \rightarrow \infty} \max \left\{\varsigma_{p}\left(\kappa_{m_{i}-1}, \kappa_{n_{i}-1}, \kappa_{n_{i}-1}\right), \frac{\varsigma_{p}\left(\kappa_{m_{i}-1}, \mathbb{Q} \kappa_{m_{i}-1}, \mathbb{Q} \kappa_{m_{i}-1}\right) \varsigma_{p}\left(\kappa_{n_{i}-1}, \mathbb{Q} \kappa_{n_{i}-1}, \mathbb{Q} \kappa_{n_{i}-1}\right)}{1+\varsigma_{p}\left(\kappa_{m_{i}-1}, \kappa_{n_{i}-1}, \kappa_{n_{i}-1}\right)}, \frac{\varsigma_{p}\left(\kappa_{m_{i}-1}, \mathbb{Q} \kappa_{m_{i}-1}, \mathbb{Q} \kappa_{m_{i}-1}\right) \varsigma_{\mathrm{p}}\left(\kappa_{n_{i}-1}, \mathbb{Q} \kappa_{n_{i}-1}, \mathbb{Q} \kappa_{n_{i}-1}\right)}{1+\varsigma_{p}\left(\mathbb{Q} \kappa_{m_{i}-1}, \mathbb{Q} \kappa_{n_{i}-1}, \mathbb{Q} \kappa_{n_{i}-1}\right)}\right\} \\
& =\limsup _{i \rightarrow \infty} \max \left\{\varsigma_{p}\left(\kappa_{m_{i}-1}, \kappa_{n_{i}-1}, \kappa_{n_{i}-1}\right), \frac{\varsigma_{p}\left(\kappa_{m_{i}-1}, \kappa_{m_{i}}, \kappa_{m_{i}}\right) \varsigma_{p}\left(\kappa_{n_{i}-1}, \kappa_{n_{i}}, \kappa_{n_{i}}\right)}{1+\varsigma_{p}\left(\kappa_{m_{i}-1}, \kappa_{n_{i}-1}, \kappa_{n_{i}-1}\right)}, \frac{\varsigma_{p}\left(\kappa_{m_{i}-1}, \kappa_{m_{i}}, \kappa_{m_{i}}\right) \varsigma_{p}\left(\kappa_{n_{i}-1}, \kappa_{n_{i}}, \kappa_{n_{i}}\right)}{1+\varsigma_{p}\left(\kappa_{m_{i}}, \kappa_{n_{i}}, \kappa_{n_{i}}\right)}\right\} \leq \varepsilon .
\end{aligned}
$$

Now, from (16) and the above inequalities, we have

$$
\begin{aligned}
\theta(\varepsilon) & \leq \theta\left(\limsup _{i \rightarrow \infty} \varsigma_{p}\left(\kappa_{m_{i}}, \kappa_{n_{i}}, \kappa_{n_{i}}\right)\right) \\
& \leq \limsup _{i \rightarrow \infty} \theta\left(M\left(\kappa_{m_{i}-1}, \kappa_{n_{i}-1}, \kappa_{n_{i}-1}\right)\right)^{\mathscr{S}\left(M\left(\kappa_{m_{i}-1}, \kappa_{n_{i}-1}, \kappa_{n_{i}-1}\right)\right)} \\
& \leq \theta(\varepsilon)^{\omega^{-1}(1)}<\theta(\varepsilon)
\end{aligned}
$$

a contradiction. Therefore, $\left\{\kappa_{n}\right\}$ is a $\varsigma_{p}$-Cauchy sequence. $\varsigma_{p}$-Completeness of $\mathcal{O}$ yields that $\left\{\kappa_{n}\right\} \varsigma_{p}$-converges to a point $\mathfrak{U} \in \mathcal{O}$.

Step III. $\mathcal{U}$ is a fixed point of $\mathbb{Q}$.

First, let $\mathbb{Q}$ is continuous, so, we have

$$
\mathfrak{U}=\lim _{n \rightarrow \infty} \kappa_{n+1}=\lim _{n \rightarrow \infty} \widehat{Q} \kappa_{n}=f \mathfrak{U} .
$$

Now, let (II) holds. Using the assumption on $\mathcal{O}$, we have $\kappa_{n} \preceq \mathfrak{U}$. Now, we show that $\mathfrak{U}=f \mathfrak{U}$. From definition of a sequential $\varsigma_{p}$-metric,

$$
\begin{aligned}
& \varsigma_{p}(\mathfrak{U}, \mathfrak{U}, f \mathfrak{U}) \leq \omega\left(\limsup _{n \rightarrow \infty} \varsigma_{p}\left(\kappa_{n+1}, \kappa_{n+1}, f \mathfrak{U}\right)\right) \\
& \leq 2 \omega\left(\theta^{-1}\left(\limsup _{n \rightarrow \infty} \theta\left(M\left(\kappa_{n}, \kappa_{n}, \mathfrak{U}\right)\right)^{\limsup _{n \rightarrow \infty}\left(M\left(\kappa_{n}, \kappa_{n}, \mathfrak{U}\right)\right)}\right)\right),
\end{aligned}
$$

where

$$
\begin{aligned}
\lim _{n \rightarrow \infty} M\left(\kappa_{n}, \kappa_{n}, \mathfrak{U}\right) \\
=\lim _{n \rightarrow \infty} \max \left\{\varsigma_{p}\left(\kappa_{n}, \kappa_{n}, \mathfrak{U}\right), \frac{\varsigma_{p}\left(\kappa_{n}, \mathscr{Q} \kappa_{n}, \mathscr{Q} \kappa_{n}\right) \varsigma_{p}\left(\kappa_{n}, \mathscr{Q} \kappa_{n}, f \mathfrak{U}\right)}{1+\varsigma_{p}\left(\kappa_{n}, \kappa_{n}, \mathfrak{U}\right)},\right. \\
\left.\quad \cdot \frac{\varsigma_{p}\left(\kappa_{n}, \mathscr{Q} \kappa_{n}, \mathscr{Q} \kappa_{n}\right) \varsigma_{p}\left(\kappa_{n}, \mathscr{Q} \kappa_{n}, f \mathfrak{U}\right)}{1+\varsigma_{p}\left(\mathscr{Q} \kappa_{n}, \mathscr{Q} \kappa_{n}, f \mathfrak{U}\right)}\right\}=0 .
\end{aligned}
$$

Therefore, we deduce that $\varsigma_{p}(\mathfrak{U}, \mathfrak{U}, f \mathfrak{U}) \leq \omega(0)=0$, so, $\mathfrak{U} \neq \mathfrak{V}$. 
Finally, suppose that the set of fixed points of $\mathbb{Q}$ be well ordered. Assume that $\mathfrak{U}$ and $\mathfrak{B}$ are two fixed points of $\mathscr{Q}$ such that $\mathfrak{U} \neq \mathfrak{B}$. Then by (16), we have

$$
\begin{aligned}
& \theta\left(\varsigma_{p}(\mathfrak{U}, \mathfrak{V}, \mathfrak{V})\right)=\theta\left(\varsigma_{p}(f \mathfrak{U}, f \mathfrak{V}, f \mathfrak{B})\right) \\
& \quad \leq \theta(M(\mathfrak{U}, \mathfrak{V}, \mathfrak{V}))^{\mathfrak{G}(M(\mathfrak{U}, \mathfrak{Q}, \mathfrak{B}))} \\
& =\theta\left(\varsigma_{p}(\mathfrak{U}, \mathfrak{V}, \mathfrak{V})\right)^{\mathscr{S}\left(\varsigma_{p}(\mathcal{U}, \mathfrak{B}, \mathfrak{B})\right)}<\theta\left(\varsigma_{p}(\mathfrak{U}, \mathfrak{V}, \mathfrak{V})\right)^{\omega^{-1}(1)} .
\end{aligned}
$$

Because

$M(\mathfrak{U}, \mathfrak{B}, \mathfrak{V})=\max \left\{\varsigma_{p}(\mathfrak{H}, \mathfrak{B}, \mathfrak{B}), \frac{\varsigma_{p}(\mathfrak{U}, \mathfrak{U}, \mathfrak{U}) \varsigma_{p}(\mathfrak{B}, \mathfrak{B}, \mathfrak{B})}{1+\varsigma_{p}(\mathfrak{U}, \mathfrak{V}, \mathfrak{B})}\right\}=\varsigma_{p}(\mathfrak{U}, \mathfrak{B}, \mathfrak{B})$.

So, we get $\ln \theta\left(\varsigma_{p}(\mathfrak{U}, \mathfrak{V}, \mathfrak{V})\right)<\omega^{-1}(1) \ln \theta\left(\varsigma_{p}(\mathfrak{U}, \mathfrak{V}, \mathfrak{V})\right)$, a contradiction. Hence $\mathfrak{U}=\mathfrak{V}$, and $\mathbb{Q}$ has a unique fixed point. Conversely, if $\mathbb{Q}$ has a unique fixed point, then the set of fixed points of $\mathbb{Q}$ is a singleton which is well ordered.

Corollary 28. Let $\left(\mathcal{O}, \varsigma_{p}, \leq\right)$ be an ordered $\varsigma_{p}$-complete SSPMS. Let $\mathbb{Q}: \mathcal{O} \rightarrow \mathcal{O}$ be an $\preceq$-increasing mapping such that $\kappa_{0} \preceq$
$\mathbb{Q}\left(\kappa_{0}\right)$ for some element $\kappa_{0} \in \mathcal{O}$. Suppose that

$$
\begin{aligned}
& \theta\left(\varsigma_{p}\left(\mathscr{Q} \kappa, \mathbb{Q} \kappa^{\prime}, \mathbb{Q} \kappa^{\prime \prime}\right)\right) \leq \theta\left(\alpha_{1} \varsigma_{p}\left(\kappa, \kappa^{\prime}, \kappa^{\prime \prime}\right)+\alpha_{2} \frac{\varsigma_{p}(\kappa, \mathbb{Q} \kappa, \mathbb{Q} \kappa) \varsigma_{p}\left(\kappa^{\prime}, \mathbb{Q} \kappa^{\prime}, \mathbb{Q} \kappa^{\prime \prime}\right)}{1+\varsigma_{p}\left(\kappa, \kappa^{\prime}, \kappa^{\prime \prime}\right)}\right.
\end{aligned}
$$

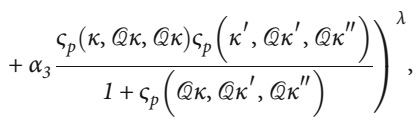

where $\alpha_{i} \in[0,1), \lambda \in(0,1)$, and $\sum_{i=1}^{3} \alpha_{i}<1$. If

(I) Q is continuous, or

(II) $(\mathcal{O}, \preceq)$ possesses the s.l.c.p

then $Q$ has a fixed point. Moreover, the set of fixed points of $\mathbb{Q}$ is well ordered if and only if $\mathbb{Q}$ has one and only one fixed point.

Definition 29. Let $\left(\mathcal{O}, \varsigma_{p}, \underline{\Omega}\right)$ is an ordered SSPMS. A mapping $\mathbb{Q}: \mathcal{O} \rightarrow \mathscr{O}$ is called a SPRGJSC of type II if there exists $\mathfrak{G} \in \mathscr{G}$ such that,

$$
\theta\left(\varsigma_{p}\left(\mathbb{Q} \kappa, Q \mathcal{Q} \kappa^{\prime}, \mathbb{Q} \kappa^{\prime \prime}\right)\right) \leq M\left(\kappa, \kappa^{\prime}, \kappa^{\prime \prime}\right)^{\mathscr{S}\left(M\left(\kappa, \kappa^{\prime}, \kappa^{\prime \prime}\right)\right)},
$$

for all comparable elements $\kappa, \kappa^{\prime}, \kappa^{\prime \prime} \in \mathcal{O}$, where

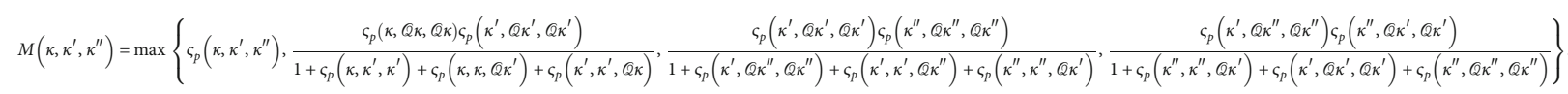

Theorem 30. Let $\left(\mathcal{O}, \varsigma_{p}, \leq\right)$ be an ordered $\varsigma_{p}$-complete SSPMS. Let $\mathbb{Q}: \mathcal{O} \rightarrow \mathcal{O}$ be a $\leq$-increasing mapping such that $\kappa_{0} \preceq \mathbb{Q}\left(\kappa_{0}\right)$ for an element $\kappa_{0} \in \mathcal{O}$. Suppose that $\mathbb{Q}$ be a SPRGJSC of type II. If

(I) Q is continuous, or

(II) $(\mathcal{O}, \preceq)$ possesses the s.l.c.p

then $\mathbb{Q}$ possesses a fixed point. Moreover, the set of fixed points of $\mathbb{Q}$ is well ordered if and only if $\mathbb{Q}$ has one and only one fixed point.

Proof. Let $\kappa_{n}=Q^{n}\left(\kappa_{0}\right)$.

Step I. We will show that $\lim _{n \rightarrow \infty} \varsigma_{p}\left(\kappa_{n}, \kappa_{n+1}, \kappa_{n+1}\right)=0$. Since $\kappa_{n}$ $\leq \kappa_{n+1}$ for each $n \in \mathbb{N}$, then by (35), we have

$$
\begin{aligned}
& \theta\left(\varsigma_{p}\left(\kappa_{n}, \kappa_{n+1}, \kappa_{n+1}\right)\right) \leq \theta\left(\varsigma_{p}\left(\kappa_{n}, \kappa_{n+1}, \kappa_{n+1}\right)\right) \\
& =\theta\left(\varsigma_{p}\left(\mathbb{Q} \kappa_{n-1}, Q \kappa_{n}, \mathbb{Q} \kappa_{n+1}\right)\right) \leq M\left(\kappa_{n-1}, \kappa_{n}, \kappa_{n+1}\right) \\
& \quad \leq \mathfrak{G}\left(\varsigma_{p}\left(\kappa_{n-1}, \kappa_{n}, \kappa_{n+1}\right)\right) \varsigma_{p}\left(\kappa_{n-1}, \kappa_{n}, \kappa_{n+1}\right)^{\mathfrak{G}\left(M\left(\kappa_{n-1}, \kappa_{n}, \kappa_{n+1}\right)\right)} \\
& \quad<\varsigma_{p}\left(\kappa_{n-1}, \kappa_{n}, \kappa_{n+1}\right) \omega^{\omega^{-1}(1)} \leq \varsigma_{p}\left(\kappa_{n-1}, \kappa_{n}, \kappa_{n+1}\right),
\end{aligned}
$$

because

$$
\begin{aligned}
& M\left(\kappa_{n-1}, \kappa_{n}, \kappa_{n}\right)=\max \left\{\varsigma_{p}\left(\kappa_{n-1}, \kappa_{n}, \kappa_{n}\right),\right. \\
& \frac{\varsigma_{p}\left(\kappa_{n-1} \mathbb{Q} \kappa_{n-1}, \mathbb{Q} \kappa_{n-1}\right) \varsigma_{p}\left(\kappa_{n}, \mathbb{Q} \kappa_{n}, \mathbb{Q} \kappa_{n}\right)}{1+\varsigma_{p}\left(\kappa_{n-1}, \kappa_{n}, \kappa_{n}\right)+\varsigma_{p}\left(\kappa_{n-1}, \kappa_{n-1}, \mathbb{Q} \kappa_{n}\right)+\varsigma_{p}\left(\kappa_{n}, \kappa_{n}, \mathbb{Q} \kappa_{n-1}\right)}, \\
& \frac{\varsigma_{p}\left(\kappa_{n}, \mathbb{Q} \kappa_{n}, \mathbb{Q} \kappa_{n}\right) \varsigma_{p}\left(\kappa_{n}, \mathbb{Q} \kappa_{n}, \mathbb{Q} \kappa_{n}\right)}{1+\varsigma_{p}\left(\kappa_{n}, \mathbb{Q} \kappa_{n}, Q \kappa_{n}\right)+\varsigma_{p}\left(\kappa_{n}, \kappa_{n}, \mathbb{Q} \kappa_{n}\right)+\varsigma_{p}\left(\kappa_{n}, \kappa_{n}, \mathbb{Q} \kappa_{n}\right)},
\end{aligned}
$$

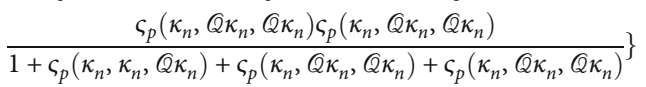

$$
\begin{aligned}
& =\max \left\{\varsigma_{p}\left(\kappa_{n-1}, \kappa_{n}, \kappa_{n}\right)\right. \text {, } \\
& \frac{\varsigma_{p}\left(\kappa_{n-1}, \kappa_{n}, \kappa_{n}\right) \varsigma_{p}\left(\kappa_{n}, \kappa_{n+1}, \kappa_{n+1}\right)}{1+\varsigma_{p}\left(\kappa_{n-1}, \kappa_{n}, \kappa_{n}\right)+\epsilon_{p}\left(\kappa_{n-1}, \kappa_{n-1}, \kappa_{n+1}\right)+\epsilon_{p}\left(\kappa_{n}, \kappa_{n}, \kappa_{n}\right)}, \\
& \frac{\varsigma_{p}\left(\kappa_{n}, \kappa_{n+1}, \kappa_{n+1}\right) \varsigma_{p}\left(\kappa_{n}, \kappa_{n+1}, \kappa_{n+1}\right)}{1+\varsigma_{p}\left(\kappa_{n}, \kappa_{n+1}, \kappa_{n+1}\right)+\varsigma_{p}\left(\kappa_{n}, \kappa_{n}, \kappa_{n+1}\right)+\varsigma_{p}\left(\kappa_{n}, \kappa_{n}, \kappa_{n+1}\right)}, \\
& \frac{\varsigma_{p}\left(\kappa_{n}, \kappa_{n+1}, \kappa_{n+1}\right) \varsigma_{p}\left(\kappa_{n}, \kappa_{n+1}, \kappa_{n+1}\right)}{1+\varsigma_{p}\left(\kappa_{n}, \kappa_{n}, \kappa_{n+1}\right)+\varsigma_{p}\left(\kappa_{n}, \kappa_{n+1}, \kappa_{n+1}\right)+\varsigma_{p}\left(\kappa_{n}, \kappa_{n+1}, \kappa_{n+1}\right)} \\
& \leq \max \left\{\varsigma_{p}\left(\kappa_{n-1}, \kappa_{n}, \kappa_{n}\right), \varsigma_{p}\left(\kappa_{n}, \kappa_{n+1}, \kappa_{n+1}\right)\right\} .
\end{aligned}
$$

Therefore, $\left\{\varsigma_{p}\left(\kappa_{n}, \kappa_{n+1}, \kappa_{n+1}\right)\right\}$ is decreasing. Similar to what have been done in Theorem 27, we have 


$$
\lim _{n \rightarrow \infty} \varsigma_{p}\left(\kappa_{n-1}, \kappa_{n}, \kappa_{n}\right)=0
$$

Step II. Now, we prove that the sequence $\left\{\kappa_{n}\right\}$ is a $\varsigma_{p}$-Cauchy sequence. In other case, i.e., that $\left\{\kappa_{n}\right\}$ is not a $\varsigma_{p}$-Cauchy sequence, for an $\varepsilon>0$, we can find two subsequences $\left\{\kappa_{m_{i}}\right\}$ and $\left\{\kappa_{n_{i}}\right\}$ of $\left\{\kappa_{n}\right\}$ such that $m_{i}$ and $n_{i}$ are the smallest index for which

$$
n_{i}>m_{i}>i \text { and } \varsigma_{p}\left(\kappa_{m_{i}}, \kappa_{n_{i}}, \kappa_{n_{i}}\right) \geq \varepsilon
$$

This means that

$$
\varsigma_{p}\left(\kappa_{m_{i}-1}, \kappa_{n_{i}-1}, \kappa_{n_{i}-1}\right)<\varepsilon
$$

From the definition of $M\left(\kappa, \kappa^{\prime}, \kappa^{\prime \prime}\right)$ and the above limits,

$$
\begin{aligned}
& \limsup M\left(\kappa_{m_{i}-1}, \kappa_{n_{i}-1}, \kappa_{n_{i}-1}\right)=\limsup \max \left\{\varsigma_{p}\left(\kappa_{m_{i}-1}, \kappa_{n_{i}-1}, \kappa_{n_{i}-1}\right),\right. \\
& \frac{\varsigma_{p}\left(\kappa_{m_{i}-1}, \mathbb{Q} \kappa_{m_{i}-1}, \mathbb{Q} \kappa_{m_{i}-1}\right) \varsigma_{p}\left(\kappa_{n_{i}-1}, \mathbb{Q} \kappa_{n_{i}-1}, \mathcal{Q} \kappa_{n_{i}-1}\right)}{1+\varsigma_{p}\left(\kappa_{m_{i}-1}, \kappa_{n_{i}-1}, \kappa_{n_{i}-1}\right)+\varsigma_{p}\left(\kappa_{m_{i}-1}, \kappa_{m_{i}-1}, \mathbb{Q} \kappa_{n_{i}-1}\right)+\varsigma_{p}\left(\kappa_{n_{i}-1}, \kappa_{n_{i}-1}, \mathbb{Q} \kappa_{m_{i}-1}\right)},
\end{aligned}
$$

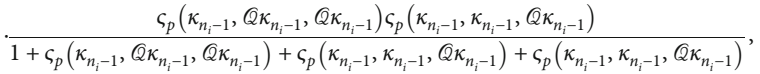

$$
\begin{aligned}
& \left.\frac{\varsigma_{p}\left(\kappa_{n_{i}-1}, \mathbb{Q} \kappa_{n_{i}-1}, \mathbb{Q} \kappa_{n_{i}-1}\right) \varsigma_{p}\left(\kappa_{n_{i}-1}, Q \mathcal{Q} \kappa_{n_{i}-1}, \mathbb{Q} \kappa_{n_{i}-1}\right)}{1+\varsigma_{p}\left(\kappa_{n_{i}-1}, \kappa_{n_{i}-1}, \mathbb{Q} \kappa_{n_{i}-1}\right)+\varsigma_{p}\left(\kappa_{n_{i}-1}, \mathbb{Q} \kappa_{n_{i}-1}, \mathbb{Q} \kappa_{n_{i}-1}\right)+\varsigma_{p}\left(\kappa_{n_{i}-1}, \mathbb{Q} \kappa_{n_{i}-1}, \mathbb{Q} \kappa_{n_{i}-1}\right)}\right\} \\
& \leq \varepsilon
\end{aligned}
$$

Now, from (35) and the above inequalities, we have

$$
\begin{aligned}
\theta(\varepsilon) & \leq \theta\left(\limsup _{i \rightarrow \infty} \varsigma_{p}\left(\kappa_{m_{i}}, \kappa_{n_{i}}, \kappa_{n_{i}}\right)\right) \\
& \leq \limsup _{i \rightarrow \infty}\left(\theta\left(M\left(\kappa_{m_{i}-1}, \kappa_{n_{i}-1}, \kappa_{n_{i}}\right)\right)^{\mathfrak{G}\left(M\left(\kappa_{m_{i}-1}, \kappa_{n_{i}-1}, \kappa_{n_{i}-1}\right)\right)}\right) \\
& \leq \theta(\varepsilon)^{\omega^{-1}(1)}
\end{aligned}
$$

which implies that $\omega^{-1}(1) \leq \limsup _{i \rightarrow \infty} \mathfrak{G}\left(M\left(\kappa_{m_{i}}, \kappa_{n_{i}-1}, \kappa_{n_{i}-1}\right.\right.$ )$)$. Now, as $\mathfrak{G} \in \mathscr{G}$, we conclude that $\left\{\kappa_{n}\right\}$ is a $\varsigma_{p}$-Cauchy sequence. $\varsigma_{p}$-Completeness of $\mathcal{O}$ yields that $\left\{\kappa_{n}\right\} \varsigma_{p}$-converges to a point $\mathfrak{U} \in \mathcal{O}$.

Step III. $\mathcal{U}$ is a fixed point of $\mathbb{Q}$.

When $Q$ is continuous, the proof is straightforward.

Now, let (II) holds. We leave the proof as it is similar to the proof of step III of Theorem 27.

Corollary 31. Let $\left(\mathcal{O}, \varsigma_{p}, \preceq\right)$ be an ordered $\varsigma_{p}$-complete SSPMS. Let $\mathbb{Q}: \mathcal{O} \rightarrow \mathcal{O}$ be $a \preceq$-increasing mapping so that $\kappa_{0} \preceq \mathbb{Q}\left(\kappa_{0}\right)$ for an element $\kappa_{0} \in \mathcal{O}$. Suppose that

$$
\begin{aligned}
& \theta\left(\varsigma_{p}\left(Q \mathcal{Q} \kappa, \mathcal{Q} \kappa^{\prime}, \mathcal{Q} \kappa^{\prime \prime}\right)\right) \\
& \leq \theta\left(\alpha_{1} \varsigma_{p}\left(\kappa, \kappa^{\prime}, \kappa^{\prime \prime}\right)+\alpha_{2} \frac{\varsigma_{p}(\kappa, Q \mathcal{Q} \kappa, \mathbb{Q} \kappa) \varsigma_{p}\left(\kappa^{\prime}, \mathbb{Q} \kappa^{\prime}, \mathbb{Q} \kappa^{\prime}\right)}{1+\varsigma_{p}\left(\kappa, \kappa^{\prime}, \kappa^{\prime}\right)+\varsigma_{p}\left(\kappa, \kappa, Q \mathcal{Q} \kappa^{\prime}\right)+\varsigma_{p}\left(\kappa^{\prime}, \kappa^{\prime}, \mathbb{Q} \kappa\right)}\right.
\end{aligned}
$$

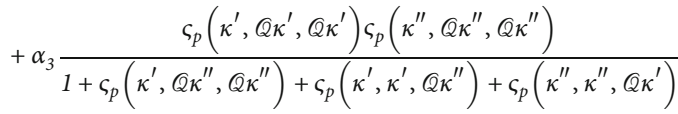

$$
\begin{aligned}
& \left.+\alpha_{4} \frac{\varsigma_{p}\left(\kappa^{\prime}, \mathbb{Q} \kappa^{\prime \prime}, \mathbb{Q} \kappa^{\prime \prime}\right) \varsigma_{p}\left(\kappa^{\prime \prime}, \mathbb{Q} \kappa^{\prime}, \mathbb{Q} \kappa^{\prime}\right)}{1+\varsigma_{p}\left(\kappa^{\prime \prime}, \kappa^{\prime \prime}, \mathbb{Q} \kappa^{\prime}\right)+\varsigma_{p}\left(\kappa^{\prime}, \mathbb{Q} \kappa^{\prime}, Q \mathcal{Q} \kappa^{\prime}\right)+\varsigma_{p}\left(\kappa^{\prime \prime}, \mathbb{Q} \kappa^{\prime \prime}, \mathbb{Q} \kappa^{\prime \prime}\right)}\right)^{\lambda}
\end{aligned}
$$

where $\alpha_{i} \in[0,1), \lambda \in(0,1)$, and $\sum_{i=1}^{4} \alpha_{i}<1$. If
(I) Q is continuous, or
(II) $(\mathcal{O}, \preceq)$ possesses the s.l.c.p

then $Q$ admits a fixed point. In addition, the set of fixed points of $Q$ is well ordered if and only if Fix $(\mathbb{Q})$ is a singleton.

The Banach contraction principle is immediately obtained from the mentioned corollaries 28, 34, and 31 .

Definition 32. Let $\left(\mathcal{O}, \varsigma_{p}, \preceq\right)$ is an ordered SSPMS. A mapping Q : $\mathcal{O} \rightarrow \mathcal{O}$ is called a SPGJSC if there exists $\mathscr{G} \in \mathscr{G}$, such that

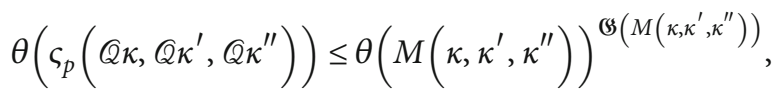

for all comparable elements $\kappa, \kappa^{\prime}, \kappa^{\prime \prime} \in \mathcal{O}$, where

$$
\begin{aligned}
& M\left(\kappa, \kappa^{\prime}, \kappa^{\prime \prime}\right)=\max \\
& \quad \cdot\left\{\varsigma_{p}\left(\kappa, \kappa^{\prime}, \kappa^{\prime \prime}\right), \varsigma_{p}\left(\kappa^{\prime}, Q \mathcal{Q} \kappa^{\prime \prime}, Q \mathcal{Q} \kappa^{\prime \prime}\right), \varsigma_{p}\left(\kappa^{\prime \prime}, Q \mathcal{Q} \kappa^{\prime}, Q \mathcal{Q} \kappa^{\prime}\right)\right\} .
\end{aligned}
$$

Theorem 33. Let $\left(\mathcal{O}, \varsigma_{p}, \preceq\right)$ be an ordered $\varsigma_{p}$-complete SSPMS. Let $\mathbb{Q}: \mathcal{O} \rightarrow \mathcal{O}$ be an $\preceq$-increasing mapping such that $\kappa_{0} \preceq Q \mathcal{Q}($ $\left.\kappa_{0}\right)$ for some element $\kappa_{0} \in \mathcal{O}$. Suppose that $\mathbb{Q}$ be a SPGJSC. If

(III) $\mathbb{Q}$ is continuous, or

(IV) $(\mathcal{O}, \preceq)$ possesses the s.l.c.p

then Q has a fixed point. Moreover, the set of fixed points of Q is well ordered if and only if Q has one and only one fixed point.

Proof. Assume that $\kappa_{n}=\mathbb{Q}^{n}\left(\kappa_{0}\right)$. Since $\kappa_{0} \preceq Q\left(\kappa_{0}\right)$ and $\mathbb{Q}$ is an $\preceq$-increasing function, we obtain by induction that

$$
\kappa_{0} \preceq Q\left(\kappa_{0}\right) \preceq Q^{2}\left(\kappa_{0}\right) \preceq \cdots \preceq Q^{n}\left(\kappa_{0}\right) \preceq Q^{n+1}\left(\kappa_{0}\right) \preceq .
$$

We shall do the proof as follows. 
Step I. We will show that $\lim _{n \rightarrow \infty} \varsigma_{p}\left(\kappa_{n}, \kappa_{n+1}, \kappa_{n+1}\right)=0$. Since $\kappa_{n}$ $\preceq \kappa_{n+1}$ for each $n \in \mathbb{N}$, then by (45), we have

$$
\begin{aligned}
\theta\left(\varsigma_{p}\left(\kappa_{n}, \kappa_{n+1}, \kappa_{n+1}\right)\right) & =\theta\left(\varsigma_{p}\left(Q \kappa_{n-1}, Q \mathcal{Q} \kappa_{n}, Q \mathcal{Q} \kappa_{n}\right)\right) \\
& \leq \theta\left(M\left(\kappa_{n-1}, \kappa_{n}, \kappa_{n}\right)\right)^{\mathfrak{G}\left(M\left(\kappa_{n-1}, \kappa_{n}, \kappa_{n}\right)\right)} \\
& \leq \theta\left(\varsigma_{p}\left(\kappa_{n-1}, \kappa_{n}, \kappa_{n}\right)\right)^{\mathfrak{G}\left(M\left(\kappa_{n-1}, \kappa_{n}, \kappa_{n}\right)\right)} \\
& <\theta\left(\varsigma_{p}\left(\kappa_{n-1}, \kappa_{n}, \kappa_{n}\right)\right)^{\omega^{-1}(1)} \\
& \leq \theta\left(\varsigma_{p}\left(\kappa_{n-1}, \kappa_{n}, \kappa_{n}\right)\right),
\end{aligned}
$$

because

$$
\begin{aligned}
M\left(\kappa_{n-1}, \kappa_{n}, \kappa_{n}\right) & =\max \left\{\varsigma_{p}\left(\kappa_{n-1}, \kappa_{n}, \kappa_{n}\right), \varsigma_{p}\left(\kappa_{n}, Q \mathcal{Q} \kappa_{n}, Q \kappa_{n}\right), \varsigma_{p}\left(\kappa_{n}, Q \kappa_{n}, Q \kappa_{n}\right)\right\} \\
& =\max \left\{\varsigma_{p}\left(\kappa_{n-1}, \kappa_{n}, \kappa_{n}\right), \varsigma_{p}\left(\kappa_{n}, \kappa_{n+1}, \kappa_{n+1}\right), \varsigma_{p}\left(\kappa_{n}, \kappa_{n+1}, \kappa_{n+1}\right)\right\} \\
& \leq \max \left\{\varsigma_{p}\left(\kappa_{n-1}, \kappa_{n}, \kappa_{n}\right), \varsigma_{p}\left(\kappa_{n}, \kappa_{n+1}, \kappa_{n+1}\right)\right\},
\end{aligned}
$$

and it is easy to see that $\max \left\{\varsigma_{p}\left(\kappa_{n-1}, \kappa_{n}, \kappa_{n}\right), \varsigma_{p}\left(\kappa_{n}\right.\right.$, $\left.\left.\kappa_{n+1}, \kappa_{n+1}\right)\right\}=\varsigma_{p}\left(\kappa_{n-1}, \kappa_{n}, \kappa_{n}\right)$, so from (48), we conclude that $\left\{\varsigma_{p}\left(\kappa_{n}, \kappa_{n+1}, \kappa_{n+1}\right)\right\}$ is decreasing. Then, there exists $r \geq 0$ such that $\lim _{n \rightarrow \infty} \varsigma_{p}\left(\kappa_{n}, \kappa_{n+1}, \kappa_{n+1}\right)=r$.

We will prove that $r=0$. This can be done as in the previous theorem. So,

$$
\lim _{n \rightarrow \infty} \varsigma_{p}\left(\kappa_{n-1}, \kappa_{n}, \kappa_{n}\right)=0,
$$

holds true.

Step II. Now, we prove that the sequence $\left\{\kappa_{n}\right\}$ is a $\varsigma_{p}$-Cauchy sequence. Suppose that $\left\{\kappa_{n}\right\}$ is not a $\varsigma_{p}$-Cauchy sequence. Then for some $\varepsilon>0$, we can find two subsequences $\left\{\kappa_{m_{i}}\right\}$ and $\left\{\kappa_{n_{i}}\right\}$ of $\left\{\kappa_{n}\right\}$ such that $m_{i}$ and $n_{i}$ are the smallest index for which

$$
n_{i}>m_{i}>i \quad \text { and } \quad \varsigma_{p}\left(\kappa_{m_{i}}, \kappa_{n_{i}}, \kappa_{n_{i}}\right) \geq \varepsilon
$$

i.e.,

$$
\varsigma_{p}\left(\kappa_{m_{i}-1}, \kappa_{n_{i}-1}, \kappa_{n_{i}-1}\right)<\varepsilon
$$

From the definition of $M\left(\kappa, \kappa^{\prime}, \kappa^{\prime \prime}\right)$ and the above limits,

$$
\begin{aligned}
& \limsup _{i \rightarrow \infty} M\left(\kappa_{m_{i}-1}, \kappa_{n_{i}-1}, \kappa_{n_{i}-1}\right)=\limsup _{i \rightarrow \infty} \max \\
& \cdot\left\{\varsigma_{p}\left(\kappa_{m_{i}-1}, \kappa_{n_{i}-1}, \kappa_{n_{i}-1}\right), \varsigma_{p}\left(\kappa_{n_{i}-1}, Q \kappa_{n_{i}-1}, Q \kappa_{n_{i}-1}\right), \varsigma_{p}\right. \\
&\left.\cdot\left(\kappa_{n_{i}-1}, Q \kappa_{n_{i}-1}, Q \kappa_{n_{i}-1}\right)\right\} \leq \varepsilon .
\end{aligned}
$$

Now, from (45) and the above inequalities, we have

$$
\begin{aligned}
\theta(\varepsilon) & \leq \theta\left(\limsup _{i \rightarrow \infty} \varsigma_{p}\left(\kappa_{m_{i}}, \kappa_{n_{i}}, \kappa_{n_{i}}\right)\right) \\
& \leq \underset{i \rightarrow \infty}{\limsup } \theta\left(M\left(\kappa_{m_{i}-1}, \kappa_{n_{i}-1}, \kappa_{n_{i}-1}\right)\right) \limsup _{i \rightarrow \infty}\left(M\left(\kappa_{m_{i}-1}, \kappa_{n_{i}-1}, \kappa_{n_{i}-1}\right)\right) \\
& \leq \theta(\varepsilon) \limsup _{i \rightarrow \infty}\left(M\left(\kappa_{m_{i}-1}, \kappa_{n_{i}-1}, \kappa_{n_{i}-1}\right)\right)
\end{aligned}
$$

which implies that $1<\underset{i \rightarrow \infty}{\limsup }\left(\mathfrak{G}\left(M\left(\kappa_{m_{i}}, \kappa_{n_{i}-1}, \kappa_{n_{i}-1}\right)\right) \leq\right.$ $\omega^{-1}(1)$. Now, as $\mathfrak{G} \in \mathscr{G}$, we conclude that $\left\{\kappa_{n}\right\}$ is a $\varsigma_{p}$-Cauchy sequence. $\varsigma_{p}$-Completeness of $\mathcal{O}$ yields that $\left\{\kappa_{n}\right\} \varsigma_{p}$-converges to a point $\mathfrak{U} \in \mathcal{O}$.

Step III. $\mathfrak{U}$ is a fixed point of $\mathbb{Q}$. This step is proved as the proof of step III of Theorem 27.

Corollary 34. Let $\left(\mathcal{O}, \varsigma_{p}, \preceq\right)$ be an ordered $\varsigma_{p}$-complete SSPMS. Let $\mathbb{Q}: \mathcal{O} \rightarrow \mathcal{O}$ be an $\preceq$-increasing mapping such that $\kappa_{0} \preceq Q($ $\left.\kappa_{0}\right)$ for an element $\kappa_{0} \in \mathcal{O}$. Suppose that

$$
\begin{aligned}
& \theta\left(\varsigma_{p}\left(\mathscr{Q} \kappa, Q \mathcal{Q} \kappa^{\prime}, \mathbb{Q} \kappa^{\prime \prime}\right)\right) \\
& \quad \leq \theta\left(\alpha_{1} \varsigma_{p}\left(\kappa, \kappa^{\prime}, \kappa^{\prime \prime}\right)+\alpha_{2} \varsigma_{p}\left(\kappa^{\prime}, \mathbb{Q} \kappa^{\prime \prime}, \mathbb{Q} \kappa^{\prime \prime}\right)+\alpha_{3} \varsigma_{p}\left(\kappa^{\prime \prime}, \mathbb{Q} \kappa^{\prime}, \mathbb{Q} \kappa^{\prime}\right)\right)^{\lambda},
\end{aligned}
$$

where $\alpha_{i} \in[0,1), \lambda \in(0,1)$ and $\sum_{i=1}^{3} \alpha_{i}<1$. If

(I) Q is continuous, or

(II) $(\mathcal{O}, \preceq)$ possesses the s.l.c.p

then Q has a fixed point. Moreover, the set of fixed points of $Q$ is well ordered if and only if Fix $(\mathbb{Q})$ is a singleton.

The Banach contraction principle is immediately obtained from the above corollary.

Example 35. Let $\mathcal{O}=[0, \infty)$ and $\varsigma_{p}$ on $\mathcal{O}$ be defined as $\varsigma_{p}(\kappa, \kappa$ $\left.{ }^{\prime}, \kappa^{\prime \prime}\right)=\left[\left|\kappa-\kappa^{\prime}\right|+\left|\kappa^{\prime}-\kappa^{\prime \prime}\right|\right]^{2}$, for all $\kappa, \kappa^{\prime}, \kappa^{\prime \prime} \in \mathcal{O}$. Let the ordering “ $\preceq$ " on $\mathcal{O}$ be as follows:

$$
\kappa \preceq \kappa^{\prime} \Leftrightarrow \kappa^{\prime} \leq \kappa, \quad \forall \kappa, \kappa^{\prime} \in \mathcal{O} .
$$

Define self-map $\mathbb{Q}$ on $\mathcal{O}$ by

$$
Q \mathcal{Q} \kappa=\frac{1}{4} \ln \left(\sqrt{\kappa^{2}+1}+\kappa\right)=\frac{1}{4} \sinh ^{-1} \kappa .
$$

Define $(\mathfrak{S}:[0,1) \rightarrow[0,1 / 2)$ as $\mathfrak{G}(t)=1 / 16 t$ and $\theta:[0, \infty)$ $\rightarrow[1, \infty)$ as $\theta(t)=\cosh t$. 
Using the mean value theorem for the function $\sinh ^{-1}$, we have

$$
\begin{aligned}
& \cosh \left(\varsigma_{p}\left(Q \kappa, Q \kappa^{\prime}, Q \kappa^{\prime \prime}\right)\right) \\
& =\cosh \left(\left|Q \kappa-Q \kappa^{\prime}\right|^{2}+\left|Q \kappa^{\prime}-Q \kappa^{\prime \prime}\right|^{2}\right) \\
& =\cosh \left(\left|\frac{1}{4} \sinh ^{-1} \kappa-\frac{1}{4} \sinh ^{-1} \kappa^{\prime}\right|^{2}+\left|\frac{1}{4} \sinh ^{-1} \kappa^{\prime}-\frac{1}{4} \sinh ^{-1} \kappa^{\prime \prime}\right|^{2}\right) \\
& \leq \cosh \left(\frac{1}{16}\left[\left|\kappa-\kappa^{\prime}\right|^{2}+\left|\kappa^{\prime}-\kappa^{\prime \prime}\right|^{2}\right]\right) \\
& \leq \cosh \left(\varsigma_{p}\left(\kappa, \kappa^{\prime}, \kappa^{\prime \prime}\right)\right)^{\mathscr{S}\left(M\left(\kappa, \kappa^{\prime}, \kappa^{\prime \prime}\right)\right)} \\
& \leq \cosh \left(M\left(\kappa, \kappa^{\prime}, \kappa^{\prime \prime}\right)\right)^{\mathfrak{G}\left(M\left(\kappa, \kappa^{\prime}, \kappa^{\prime \prime}\right)\right)} .
\end{aligned}
$$

Note that for all $x \geq 0$ one can see that $\cosh ((1 / 16) x) \leq$ $\cosh (x)^{(1 / 16) x}$. Thus, (16) is satisfied for all $\kappa, \kappa^{\prime}, \kappa^{\prime \prime} \in \mathcal{O}$. Therefore, all the conditions of Theorem 27 hold true. Moreover, 0 is a fixed point of $Q$.

\section{Existence of a Solution for an Integral Equation}

We consider the following integral equation:

$$
\kappa(t)=\int_{a}^{b} \Gamma(t, s, \kappa(s)) d s+\gamma(t)
$$

where $b>a \geq 0$. Our aim in this part is to present the existence of a solution for (59) which is an element of $\mathcal{O}=C[a$, $b]$ as an application of Theorem 30 .

The above equation can be changed as follows.

Let $\mathbb{Q}: \mathcal{O} \rightarrow \mathcal{O}$ be defined by

$$
\mathcal{Q} \kappa(t)=\int_{a}^{b} \Gamma(t, s, \kappa(s)) d s+\gamma(t)
$$

for all $\kappa \in \mathcal{O}$ and for all $t \in[a, b]$. Clearly, existence of a solution for (59) is equivalent to the existence of a fixed point of Q.

Let

$$
d(\mathcal{U}, \mathscr{V})=\max _{t \in a, b]}|\mathcal{U}(t)-\mathscr{V}(t)|^{p}=\|\mathscr{U}-\mathscr{V}\|_{\infty}^{p} .
$$

Let $\mathcal{O}$ be equipped with the sequential $\varsigma_{p}$-metric given by

$$
\varsigma_{p}(\mathscr{U}, \mathscr{V}, \mathscr{W})=\mathrm{F}(d(\mathscr{U}, \mathscr{V})+d(\mathscr{V}, \mathscr{W})),
$$

for all $\mathscr{U}, \mathscr{V}, \mathscr{W} \in \mathcal{O}$ where $\mathrm{F}:[0, \infty) \rightarrow[0, \infty)$ is a strictly increasing continuous function with $t \leq \mathrm{F}(t)$ for $t \geq 0$ and $\mathrm{F}(0)=0$ which is a $\varsigma_{p}$-complete SSPMS with control function $\omega(t)=\mathrm{F}\left(2^{p-1} t\right)$. Let the partial ordered $\preceq$ given by $\kappa$ $\preceq \kappa^{\prime} \Leftrightarrow \kappa(t) \leq \kappa^{\prime}(t)$, for all $t \in[a, b]$, be defined on $\mathcal{O}$. ( $\mathcal{O}$,

$\preceq)$ has the sequential limit comparison property [17].

Now, we state the following consequence.
Theorem 36. Suppose that

(i) $\Gamma:[a, b] \times[a, b] \times R \rightarrow R \quad$ and $\quad \gamma:[a, b] \rightarrow R$ are continuous

(ii) $\left|\Gamma(t, r, \kappa(r))-\Gamma\left(t, r, \kappa^{\prime}(r)\right)\right| \leq\left|\kappa(r)-\kappa^{\prime}(r)\right|$

for all $t, r \in a, b]$ and for all $\kappa, \kappa^{\prime} \in \mathcal{O}$ with $\kappa \preceq \kappa^{\prime}$;

(iii) for all positive values $\lambda, \mu, v$ we have

$$
\theta(\mathrm{F}(\lambda \mu+\lambda v)) \leq \sqrt{\theta(\mathrm{F}(\mu+v))}
$$

(iv) for some function $\alpha:[a, b] \rightarrow \mathbb{R}$ one has

$$
\alpha(t) \leq \int_{a}^{b} \Gamma(t, s, \alpha(s)) d s+\gamma(t) .
$$

Then, the integral equations (59) has a solution $\kappa \in \mathcal{O}$.

Proof. Let $\kappa, \kappa^{\prime} \in \mathcal{O}$ be such that $\kappa \succeq \kappa^{\prime}$. From condition (ii), for all $t \in[a, b]$, we have

$$
\begin{aligned}
& \theta\left(\mathrm{F}\left(\left|\mathcal{Q} \kappa(t)-\mathcal{Q} \kappa^{\prime}(t)\right|^{p}+\left|\mathcal{Q} \kappa^{\prime}(t)-Q \bar{Q} \kappa^{\prime \prime}(t)\right|^{p}\right)\right) \\
& \leq \theta\left(\mathrm { F } \left(\left(\int_{a}^{b}\left|\Gamma(t, s, \kappa(s))-\Gamma\left(t, s, \kappa^{\prime}(s)\right)\right| d s\right)^{p}\right.\right. \\
& \left.\left.+\left(\int_{a}^{b}\left|\Gamma\left(t, s, \kappa^{\prime}(s)\right)-\Gamma\left(t, s, \kappa^{\prime \prime}(s)\right)\right| d s\right)^{p}\right)\right) \\
& \leq \theta\left(\mathrm { F } \left((a-b)^{\frac{p}{q}} \int_{a}^{b}\left|\Gamma(t, s, \kappa(s))-\Gamma\left(t, s, \kappa^{\prime}(s)\right)\right|^{p} d s\right.\right. \\
& \left.\left.+(a-b)^{\frac{p}{q}} \int_{a}^{b}\left|\Gamma\left(t, s, \kappa^{\prime}(s)\right)-\Gamma\left(t, s, \kappa^{\prime \prime}(s)\right)\right|^{p} d s s s\right)\right) \\
& \leq \theta\left(\mathrm { F } \left((a-b)^{\frac{p}{q}} \int_{a}^{b}\left|\kappa(s)-\kappa^{\prime}(s)\right|^{p} d s\right.\right. \\
& \left.\left.+(a-b)^{\frac{p}{q}} \int_{a}^{b}\left|\kappa^{\prime}(s)-\kappa^{\prime \prime}(s)\right|_{\infty}^{p} d s\right)\right) \\
& \leq \theta\left(\mathrm{F}\left((a-b)^{\frac{2 p}{q}}\left\|\kappa-\kappa^{\prime}\right\|_{\infty}^{p}+(a-b)^{\frac{2 p}{q}}\left\|\kappa^{\prime}-\kappa^{\prime \prime}\right\|^{p}\right)\right) \\
& \leq \sqrt{\theta\left(\mathrm{F}\left(\left\|\kappa-\kappa^{\prime}\right\|_{\infty}^{p}+\left\|\kappa^{\prime}-\kappa^{\prime \prime}\right\|^{p}\right)\right)} \text {. }
\end{aligned}
$$

Therefore, taking the sup on $[a, b]$, we have

$$
\theta\left(\varsigma_{p}\left(\mathscr{Q} \kappa, Q \kappa^{\prime}, Q \kappa^{\prime \prime}\right)\right) \leq \sqrt{\theta\left(M\left(\kappa, \kappa^{\prime}, \kappa^{\prime \prime}\right)\right)},
$$


where

$$
\begin{aligned}
& M\left(\kappa, \kappa^{\prime}, \kappa^{\prime \prime}\right)=\max \left\{\varsigma_{p}\left(\kappa, \kappa^{\prime}, \kappa^{\prime \prime}\right), \frac{\varsigma_{p}(\kappa, \kappa, Q \mathcal{Q} \kappa) \varsigma_{p}\left(\kappa^{\prime}, \kappa^{\prime}, \mathbb{Q} \kappa^{\prime}\right)}{1+\mathrm{F}\left[\varsigma_{p}\left(\kappa, \kappa^{\prime}, \kappa^{\prime}\right)+\varsigma_{p}\left(\kappa, \kappa, Q \mathcal{Q} \kappa^{\prime}\right)\right]},\right. \\
& \cdot \frac{\varsigma_{p}\left(\kappa^{\prime}, \kappa^{\prime}, Q \kappa^{\prime}\right) \varsigma_{p}\left(\kappa^{\prime \prime}, \kappa^{\prime \prime}, Q \kappa^{\prime \prime}\right)}{1+\mathrm{F}\left[\varsigma_{p}\left(\kappa^{\prime}, \kappa^{\prime \prime}, \kappa^{\prime \prime}\right)+\varsigma_{p}\left(\kappa^{\prime}, \kappa^{\prime}, Q \mathcal{Q} \kappa^{\prime \prime}\right)\right]}, \\
& \left.\frac{\varsigma_{p}\left(\kappa^{\prime}, \kappa^{\prime \prime}, \kappa^{\prime \prime}\right) \varsigma_{p}\left(\kappa^{\prime}, \kappa^{\prime}, \kappa^{\prime \prime}\right)}{1+\varsigma_{p}\left(\kappa^{\prime}, \mathscr{Q} \kappa^{\prime}, Q \mathcal{Q} \kappa^{\prime}\right)+\varsigma_{p}\left(\kappa^{\prime \prime}, \mathbb{Q} \kappa^{\prime \prime}, \mathbb{Q} \kappa^{\prime \prime}\right)}\right\} \text {, }
\end{aligned}
$$

So, from Theorem 30 , there exists $\kappa \in \mathcal{O}$, a fixed point of $\mathcal{Q}$ which is a solution of (59).

\section{Conclusions}

In this paper, we worked on a space which fails the commutativity property, usual rectangular property, and continuous property. So, we are very restricted in such spaces.

\section{Data Availability}

No data were used to support this study.

\section{Conflicts of Interest}

The authors declare that there is not any competing interest regarding the publication of this manuscript.

\section{Authors' Contributions}

All authors contributed equally to the writing of this paper. All authors read and approved the final manuscript.

\section{References}

[1] S. Czerwik, "Contraction mappings in $b$-metric spaces," Acta Mathematica et Informatica Universitatis Ostraviensis, vol. 1, pp. 5-11, 1993.

[2] V. Parvaneh and Z. Kadelburg, "Extended partial $b$-metric spaces and some fixed point results," Filomat, vol. 32, no. 8, pp. 2837-2850, 2018.

[3] V. Parvaneh and S. J. H. Ghoncheh, "Fixed points of $(\Psi, \Phi)_{\Omega}$ -contractive mappings in ordered $P$-metric spaces," Global Analysis and Discrete Mathematics, vol. 4, no. 1, pp. 15-29, 2020.

[4] M. Jleli and B. Samet, "A generalized metric space and related fixed point theorems," Fixed point Theory and Applications, vol. 2015, no. 1, Article ID 61, 2015.

[5] Z. Ma, L. Jiang, and H. Sun, " $C *$-algebra-valued metric spaces and related fixed theorems," Fixed Point Theory and Aplications, vol. 2014, no. 1, article 206, 2014.

[6] N. Souayaha and N. Mlaikib, "A fixed point theorem in $S_{b}$ -metric spaces," Journal of Mathematics and Computer Science, vol. 16, no. 2, pp. 131-139, 2016.
[7] S. Sedghi, A. Gholidahneh, and K. P. R. Rao, "Common fixed point of two $R$-weakly commuting mappings in $S_{b}$-metric spaces," Mathematical Sciences Letters, vol. 6, no. 3, pp. 249253, 2017.

[8] Z. Mustafa, R. J. Shahkoohi, V. Parvaneh, Z. Kadelburg, and M. M. M. Jaradat, "Ordered $S_{p}$-metric spaces and some fixed point theorems for contractive mappings with application to periodic boundary value problems," Fixed Point Theory and Aplications, vol. 2019, no. 1, article 16, 2019.

[9] Z. Mustafa and B. Sims, "A new approach to generalized metric spaces," Journal of Nonlinear and Convex Analysis, vol. 7, pp. 289-297, 2006.

[10] J. Rezaei Roshan, V. Parvaneh, S. Sedghi, N. Shobkolaei, and W. Shatanawi, "Common fixed points of almost generalized $(\psi, \varphi)_{s}$-contractive mappings in ordered $b$-metric spaces," Fixed Point Theory and Applications, vol. 2013, no. 1, Article ID 159, 2013.

[11] R. J. Shahkoohi and A. Razani, "Some fixed point theorems for rational Geraghty contractive mappings in ordered b-metric spaces," Journal of Inequalities and Applications, vol. 2014, no. 1, Article ID 373, 2014.

[12] K. Roy, I. Beg, and M. Saha, "Sequentially compact $S^{J S}$-metric spaces," Commun. Optin. Theory, vol. 2020, article 4, pp. 1-7, 2020.

[13] K. Roy, S. Panja, M. Saha, and V. Parvaneh, "An extended $b$ -metric-type space and related fixed point theorems with an application to nonlinear integral equations," Advances in Mathematical Physics, vol. 2020, Article ID 8868043, 7 pages, 2020.

[14] S. Sedghi, N. Shobe, and A. Aliouche, "A generalization of fixed point theorem in S-metric spaces," Matematichki Vesnik, vol. 64, no. 249, pp. 258-266, 2012.

[15] Z. Mustafa, J. R. Roshan, and V. Parvaneh, "Coupled coincidence point results for $(\psi, \varphi)$-weakly contractive mappings in partially ordered $G_{b}$-metric spaces," Fixed Point Theory and Applications, vol. 2013, no. 1, Article ID 206, 2013.

[16] M. Jleli, E. Karapinar, and B. Samet, "Further generalizations of the Banach contraction principle," Journal of Inequalities and Applications, vol. 2014, no. 1, Article ID 439, 2014.

[17] J. J. Nieto and R. Rodríguez-López, "Existence and uniqueness of fixed point in partially ordered sets and applications to ordinary differential equations," Acta Mathematica Sinica, English Series, vol. 23, no. 12, pp. 2205-2212, 2007. 